\title{
Comprehensive Stiffness of Prestressed Lattice Materials
}

\author{
Mostafa S. A. Elsayed \\ Department of Mechanical Engineering, McGill University \\ Macdonald Engineering Building, Room 372 \\ 817 Sherbrooke St West, Montreal, Quebec H3A 2K6, Canada \\ Tel.: 1-514-398-6295Ｅ-mail: mostafa.elsayed@mail.mcgill.ca \\ Damiano Pasini (Corresponding author) \\ Department of Mechanical Engineering, McGill University \\ Macdonald Engineering Building, Room 372 \\ 817 Sherbrooke St West, Montreal, Quebec H3A 2K6, Canada \\ Tel: 1-514-398-6295Ｅ-mail: damiano.pasini@mcgill.ca
}

Received: January 13, 2012 Accepted: February 8, 2012 Published: Apri1 1, 2012

doi:10.5539/jmsr.v1n2p87

URL: http://dx.doi.org/10.5539/jmsr.v1n2p87

\begin{abstract}
Several approaches to obtain the comprehensive stiffness of finite frameworks are present in literature; yet, the formulation has not been addressed for lattice materials and infinitely periodic structures. The objective of this paper is to introduce a systematic method to calculate the comprehensive stiffness of prestressed, infinitely periodic, structures and lattice materials with pin- and rigid-jointed connectivity. We first derive the comprehensive stiffness of a finite framework through the superposition of its material and nonlinear geometrical stiffness. By using the Bloch's theorem, we derive the irreducible form of the stiffness system of the finite framework, which represents the stiffness behaviour of the corresponding infinite, periodic assembly. Finally, the comprehensive stiffness of the infinite lattice is homogenized to generate the stiffness characteristics of the lattice material. A detailed example is provided to show the application of the methodology. Closed-form expressions of the elastic properties are presented for 12 planar lattices.
\end{abstract}

Keywords: Geometrical stiffness, Comprehensive stiffness, Tangent stiffness, Periodic structures, Lattice materials

\section{Introduction}

Material and geometric nonlinearities are commonly encountered in structural analysis. The former is developed due to the nonlinear elastic, plastic and/or viscoelastic behaviour of the material. The latter starts once the structural deformations exceed the infinitesimal limit at which strains are sufficiently large to generate a change in the initial configuration of the structure. In this case, the equilibrium system of the deformed structure is not equivalent to its state in the undeformed configuration. As a result, the equilibrium state should be formulated in the deformed configuration to factor in an additional term, namely the geometrical stiffness.

Geometrical nonlinearities are normally present in the response of any structure. In the context of small strain theory, their effect is often negligible; on the other hand, their influence cannot be ignored in kinematically indeterminate frameworks. A kinematically indeterminate framework is a pin-jointed structure with internal mechanisms, i.e. the modes of structural deformations occur without element deformation. Some of these internal mechanisms generate finite displacements at each external loading increment; other mechanisms, on the other hand, are exceptional as the structure encounters only first order infinitesimal mechanisms accompanied by higher order strains. Such kinds of structures are prestressable and can be found in several natural and engineering systems such as cable systems (e.g. cable nets), tensegrity frameworks, pneumatic domes and fabric roofs (Kuznetsov, 1997; Tilbert, 2002).

The static analysis of prestressed structures finds its roots in the works of Mohr (1885), Maxwell (1890), 
Levi-Civita and Amaldi (1930); and more recently in the contributions of Calladine (1982), Calladine and Pellegrino (1986; 1991), Volokh and Vilnay (1997) and Kuznetsov (1997; 2000). Maxwell (1890) first identified the minimum number of bars for a pin-jointed framework to be kinematically and statically determinate, i.e. "simply stiff". If the number of bars is below the minimum condition of Maxwell, the structure is a mechanism, unless its nodes are fixed. In this case, the mechanical behaviour of the framework is dominated by the bending failure of its constituents. On the other hand, if the number of bars is above the minimum condition of Maxwell, then the structure is "over-stiff", for it has a redundant number of bars. The failure modes of simply stiff and over-stiff frameworks are dominated by the axial stress of its constituents, which results in a stretching dominated behaviour. Calladine (1978), Pellegrino (1993) and Pellegrino and Calladine (1986) reviewed the linear-algebraic basis of Maxwell's rule. Their formulation generalized Maxwell's rule to obtain the states of self-stresses and the states of internal mechanisms from the fundamental subspaces of the equilibrium and the kinematic matrices of a pin-jointed framework. The generalized Maxwell's rule can be used to obtain an accurate prediction of the determinacy state of finite structures. Deshpande et al. (2001a) extended the determinacy analysis to account for infinitely periodic frameworks. In their work, the mechanics of a limited set of pin-jointed lattice topologies with infinite periodicity was examined. In particular, only topologies wherein the nodes are similarly-situated, i.e. the framework appears the same and in the same orientation regardless of the viewpoint, were studied. In 2D, these are the regular square and triangular lattices; in $3 \mathrm{D}$, this set includes the regular octet-truss. The generalized Maxwell's rule was used to prove that the necessary but not sufficient nodal connectivity, $Z$, of a structure to be stretching dominated is $Z=4$ in $2 \mathrm{D}$ and $Z=6$ in $3 \mathrm{D}$. On the other hand, the sufficient nodal connectivity was proven to be $Z=6$ in $2 \mathrm{D}$ and $Z=12$ in 3D. More recently, Hutchinson (2004) used the Bloch's theorem for modeling periodic waves in an infinite lattice structure. Following this approach, Elsayed and Pasini (2010a; 2010b) developed a matrix-based methodology to analyze the determinacy state of infinite lattice structures with an arbitrary cell topology and obtain the homogenized material stiffness properties of both pin-jointed and rigid-jointed infinitely periodic frameworks.

From the aforementioned works, it appears that the analysis of the determinacy state of finite and infinite frameworks is well established. However, the impact of the determinacy state on the static response of the structures in the form of comprehensive stiffness characteristics has been investigated only for finite frameworks. As demonstrated in literature, the geometrical stiffness can play a major role in the comprehensive stiffness response of a prestressed structure. In this paper, we extend the literature on this topic to model the comprehensive stiffness of infinite periodic structures. We generalize their stiffness characteristics to account for the geometrical non-linearity of periodic frameworks. The homogenized, comprehensive stiffness of periodic cellular materials are for the first time provided for 12 lattice topologies. The role that the geometrical stiffness plays in the static response of a given lattice is discussed.

The paper is organized in five sections. After the introduction, the mathematical description and the model of the comprehensive stiffness are presented for pin- and rigid-jointed frameworks as well as for lattice materials. To demonstrate the steps of the methodology, the procedure is applied in section 3 to the Kagome lattice material. The fourth section discusses the impact of the comprehensive stiffness on the stiffness resistance of two selected cell topologies, the Kagome and the $3^{3} \cdot 4^{2}$ lattices. Concluding remarks are given in the last section.

\section{Analysis}

A periodic structure can be generated by tessellating a unit cell along periodic vectors. To characterize the physical properties of an infinite lattice, it is common to study the attributes of the unit module, which is considered as the Representative Volume Element (Hill, 1963; Shan \& Gokhale, 2002). The unit cell can be described by the envelope of the unit cell and its bases. The former governs the structure periodicity, described by the translational symmetry bases, $\overrightarrow{a_{k}}$, where $k \in\{1, . ., n\}$ with $n=2$ in $2 \mathrm{D}$ and $n=3$ in 3D. The latter representing the physical structure contains two groups, namely, the node bases group and the bar bases group. Fig (1) illustrates this concept applied to the Kagome lattice. Figure (1a) shows the structure of a cluster of unit cells of the lattice. 


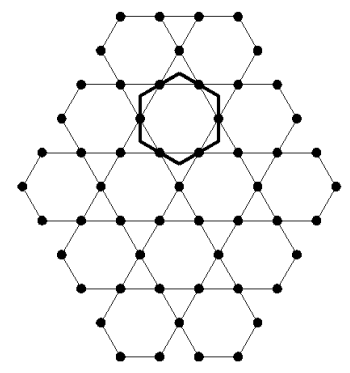

(a)

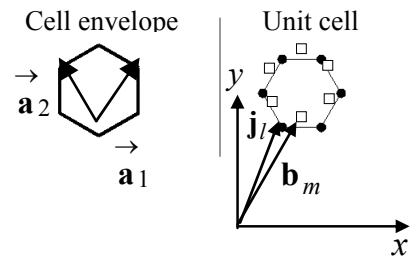

(b) (c)

口

Geometric centroid of a bar... Node

Figure 1. Kagome lattice structure

Figure (1b) shows the cell envelope along with the translational primitive bases $a_{1}$ and $a_{2}$. Figure (1c) illustrates the bar position vectors, $b_{m}$, and the node position vectors, $j_{l}$, of a unit cell, where $m \in\{1,2, \ldots b\}$ and $l \in\{1,2, \ldots j\}, b$ and $j$ are, respectively, the total number of bars and the total number of nodes within the unit cell.

In the next section, we present the comprehensive stiffness system of the unit cell and we extend its formulation to the infinite lattice. For simplification, we assume the cell elements have the same cross-sectional area and material properties.

\subsection{Stiffness System of the Unit Cell as a Finite Structure}

The stiffness system of a framework with $b$ elements connected between $j$ nodes is given by:

$$
\mathbf{K d}=\mathbf{f}
$$

where $\mathbf{K} \in R^{n j \times n j}$ is the stiffness matrix that relates the nodal deformation vector, $\mathbf{d} \in R^{n j}$, of the structure to its nodal force vector, $\mathbf{f} \in R^{n j}$ (McCormac, 2006), with $\mathrm{n}=2$ and $\mathrm{n}=3$ for pin- and rigid-jointed assemblies. For a prestressed, kinematically and statically indeterminate, structure in which finite deformations are expected, the equation of the force equilibrium must be formulated in the deformed configuration. Eqn (1) is then modified to account for the geometrical stiffness as:

$$
\left(\mathbf{K}_{E}+\mathbf{K}_{G}\right) \mathbf{d}=\mathbf{f}
$$

where $\mathbf{K}_{E} \in R^{n j \times n j}$ and $\mathbf{K}_{G} \in R^{n j \times n j}$ are respectively the material and the geometrical stiffness matrices. To easy the formulation of the comprehensive stiffness for pin- and rigid-jointed finite frameworks, we rewrite eqn (2) as:

$$
\begin{aligned}
& \left({ }_{p} \mathbf{K}_{C}\right)_{p} \mathbf{d}=\left\{{ }_{p} \mathbf{K}_{E}^{\text {bar }}{ }_{{ }_{p}} \mathbf{K}_{G}^{\text {bar }}\right\}_{p} \mathbf{d}={ }_{p} \mathbf{f} \\
& \left({ }_{r} \mathbf{K}_{C}\right)_{r} \mathbf{d}=\left\{{ }_{r} \mathbf{K}_{E}^{\text {bar }}+{ }_{r} \mathbf{K}_{E}^{\text {beam }}+_{r} \mathbf{K}_{G}^{\text {frame }}\right\}_{r} \mathbf{d}={ }_{r} \mathbf{f}
\end{aligned}
$$

where subscripts $p$ and $r$, refer, respectively, to the attributes of pin-jointed and rigid-jointed structures; subscripts $E$ and $\mathrm{G}$ refer, respectively, to material and geometrical stiffness characteristics. Since the geometrical stiffness term in equation (3) is a function of the axial forces of the structural elements, we present in the next section the formulation of the axial forces in statically and kinematically indeterminate frames.

\subsubsection{Tension Forces in the elements of the Unit Cell}

The equilibrium and kinematic systems of a pin-jointed structure with $b$ elements connected between $\mathrm{j}$ nodes can be expressed as:

$$
\begin{aligned}
\mathbf{A t} & =\mathbf{f} \\
\mathbf{B d} & =\mathbf{e}
\end{aligned}
$$

where $\mathbf{A} \in R^{2 j \times b}$ and $\mathbf{B} \in R^{b \times 2 j}$ are Jacobian matrices with entries of direction cosines that transform, respectively, the vector of tension forces of the structural elements, $\mathbf{t} \in R^{b}$, to the vector of the nodal forces, 
$\mathbf{f} \in R^{2 j}$, and the vector of nodal displacements, $\mathbf{d} \in R^{2 j}$, to the vector of element deformations, $\mathbf{e} \in R^{b}$, (Kuznetsov, 1997; 2000).

The vector of tension forces of the structural elements, $t$, is composed of two terms: (i) $\mathbf{t}_{f} \in R^{b}$, depends on the applied external loading, f, and (ii) $\mathbf{s}_{G}$, generated by the elongations imposed in the redundant elements of the structure. Both terms contribute to the structural geometrical stiffness. Thus:

$$
\mathbf{t}=\mathbf{t}_{f}+\mathbf{S} \boldsymbol{\gamma}=\mathbf{t}_{f}+\mathbf{s}_{G}
$$

where $\mathbf{S} \in R^{b \times s}$ is the matrix of states of self-stresses formulated by concatenating the modes of states of self-stress into its columns; $\mathrm{s}$ is the number of states of self-stress in the structure. $\gamma \in R^{s}$ is a vector of arbitrary constants that are used to generate a linear combination of the independent modes of the states of self-stress.

Thus, a global state of self-stress, $\mathbf{s}_{G}$, which describes the modes of axial forces induced in a framework with no applied external load, can be expressed as:

$$
\mathbf{s}_{G}=\mathbf{S} \boldsymbol{\gamma}
$$

The states of self-stress are the non-pivotal modes in the column space of the equilibrium matrix, A. They can be obtained by evaluating the reduced row echelon form (Strang, 1998) of the equilibrium matrix and calculating the non-pivotal modes in its column space.

The element deformation vector, $\mathbf{e} \in R^{b}$, on the other hand, can be expressed as:

$$
\mathbf{e}=\mathbf{e}_{0}+\mathbf{F t}
$$

where $\mathbf{e}_{0} \in R^{b}$ is the vector of imposed elongations in the redundant elements of the structure due to heating, misfit or by prestressing a member through the use of, for example, smart materials, (Sener, et.al., 1994). $\mathbf{F} \in R^{b \times b}$ in eqn. (8) is a compliance diagonal matrix with entries expressed by:

$$
f_{k k}=\frac{l_{k}}{E A}
$$

The independent modes of mechanisms of a pin-jointed structure are found in the column space of the kinematic matrix, B, and are computed following the same approach as for the states of self-stress. In the current analysis, the modes of mechanisms encountered in a structure include both the rigid-body motions and the states of internal mechanisms. The reason for the occurrence of rigid-body motions in the unit cell is that its structure is not constrained into a foundation. It is worth mentioning here that $\mathrm{B}=\mathrm{A}^{\mathrm{T}}$, where $\mathrm{A}^{\mathrm{T}}$ is the transpose of the equilibrium matrix A, which is obtained through the principle of virtual work (Timoshenko \& Young, 1945). This equivalence indicates that the modes of mechanisms are also found in the row space of the equilibrium matrix and can be determined by computing the left-null space of the equilibrium matrix.

Pellegrino $(1992 ; 1990 ; 1988)$, classified pin-jointed structures based on the number of states of self-stress, s, and the number of states of internal mechanisms, m, as shown in Table (1). Guest and Hutchinson (2003), demonstrated that periodic lattice structures cannot be simultaneously statically and kinematically determinate; thus the structural type I in Table (1) is excluded from this study. On the other hand, the pretension of an element $(\mathrm{s}>0)$ can significantly increase the stiffness resistance of the framework. In this paper, we examine structures of types III and IV (Table (1)). In particular, we investigate the effect of the term $\mathbf{s}_{G}$ of the geometrical stiffness on the stiffness characteristics offered by lattice materials and periodic structures, regardless of the external loading.

Table 1. Classification of Structural Assemblies

\begin{tabular}{|c|c|c|c|}
\hline Type & $\begin{array}{c}\text { \# of modes of states of } \\
\text { self-stress (s) }\end{array}$ & $\begin{array}{c}\text { \# of modes of states of internal } \\
\text { mechanisms }(m)\end{array}$ & Static and kinematic properties \\
\hline I & $s=0$ & $m=0$ & Statically and kinematically determinate \\
\hline II & $s=0$ & $m>0$ & $\begin{array}{c}\text { Statically determinate and kinematically } \\
\text { indeterminate }\end{array}$ \\
\hline III & $s>0$ & $m=0$ & $\begin{array}{c}\text { Statically indeterminate and } \\
\text { kinematically determinate }\end{array}$ \\
\hline IV & $s>0$ & $m>0$ & $\begin{array}{c}\text { Statically and kinematically } \\
\text { indeterminate }\end{array}$ \\
\hline
\end{tabular}


Of course once the applied external loading, expressed in the form of macroscopic stress or strain fields, is determined, the term of the axial forces, $\mathbf{t}_{f}$, can be obtained and a similar strategy can be followed to compute the resulting geometrical stiffness.

\subsubsection{Global State of Self-Stress}

The computation of the global state of self-stress $\mathbf{s}_{G}$ is performed in two steps. The first is to compute the matrix S of the states of self-stress, as described in the previous section. The second is to determine the vector of arbitrary constants, $\gamma$, as a function of the imposed elongations, $\mathbf{e}_{0}$. We use the orthogonality condition to obtain the vector of arbitrary constants that generates a linear combination of the states of self-stress. Following the approach of Pellegrino $(1990 ; 1993)$, to impose the vector of element deformations, $\mathbf{e}$, to be orthogonal to the null space of the equilibrium matrix, $\mathbf{A}$, we write:

$$
\mathbf{S}^{T} \mathbf{e}=0
$$

Substituting eqn (6) into eqn (8), then substituting the result into eqn (10) and rearranging, gives:

$$
\boldsymbol{\gamma}=-\left(\mathbf{S}^{T} \mathbf{F S}\right)^{-1} \mathbf{S}^{T}\left(\mathbf{e}_{0}+\mathbf{F} \mathbf{t}_{f}\right)
$$

Similar formulations to the orthogonality condition of eqn (11) can be found in textbooks on matrix analysis of structures (Livesley, 1975; Pestel \& Leckie, 1963).

Since we are interested in axial forces excited by the imposed elongations, $\mathbf{e}_{0}$, by setting $\mathbf{t}_{f}=0$ in eqn (11) we obtain:

$$
\gamma=-\left(\mathbf{S}^{T} \mathbf{F S}\right)^{-1} \mathbf{S}^{T} \mathbf{e}_{0}
$$

Substituting eqn (12) into eqn (7) results in the vector of global state of self-stress:

$$
\mathbf{s}_{G}=-\mathbf{S}\left(\mathbf{S}^{T} \mathbf{F S}\right)^{-1} \mathbf{S}^{T} \mathbf{e}_{0}
$$

The vector of imposed elongations $\mathbf{e}_{0} \in R^{b}$ can be formulated as:

$$
\mathbf{e}_{0}=\varepsilon_{0} \mathbf{e}_{l}
$$

$\varepsilon_{0}$ is a scalar corresponding to the nominal strain assumed in the redundant elements. $\varepsilon_{0}$ is used to control the level of pre-tension field within the lattice; $\mathbf{e}_{l} \in R^{b}$ is a vector with entries corresponding to the redundant elements in the unit cell expressed as $\left(e_{l}\right)_{i}=l_{i} ; l_{i}$ is the length of the $i^{\text {th }}$ redundant element within the unit cell and $i \in\{1, . ., r\}$ and the $r$ 's are indices of the redundant elements in the unit cell. Other indices corresponding to pivotal elements in the equilibrium matrix are set equal to zero in the $\mathbf{e}_{l} \in R^{b}$ vector.

Substituting eqn (14) into eqn (13) results in:

$$
\mathbf{s}_{G}=-\left(\varepsilon_{0}\right) \mathbf{S}\left(\mathbf{S}^{T} \mathbf{F S}\right)^{-1} \mathbf{S}^{T} \mathbf{e}_{l}
$$

The axial forces in the elements computed by $\mathbf{s}_{G}$ are substituted into eqns (3) to obtain the comprehensive stiffness of the structure.

\subsection{Stiffness System of the Infinite Lattice Structure}

In this section, we extend the computation of the stiffness system of finite structures to infinite periodic structures. To compute the geometrical stiffness of the infinite periodic structure, we derive the states of self-stress that represent the irreducible expressions of their counterparts in the infinite lattice. The Bloch's theorem is used to compute the irreducible forms of the static wave-functions which are employed to determine the equilibrium system of the infinite lattice. The reduced equilibrium matrix, $\widetilde{\mathbf{A}}$, is computed at the different wave-numbers that are derived from the irreducible first Brillouin zone of the reciprocal lattice (Brillouin, 1946). The null space of the matrix $\widetilde{\mathbf{A}}$ is evaluated at the different wave-numbers. The independent vectors developed from the null space of the reduced equilibrium matrix represent the modes of states of self-stress of the infinite lattice. These modes are concatenated as the columns of the matrix $\widetilde{\mathbf{S}}$ of the reduced states of self-stress. 
It should be noted that at the wave-number $\omega=(0,0)$ the row space of the reduced equilibrium matrix $\widetilde{\mathbf{A}}$ contains two kinematical modes of translational rigid-body motion as the lattice structure is not constrained into a foundation. A rigid-body rotation is constrained due to the basic assumption of the translational periodicity (Guest \& Hutchinson, 2003). An accurate computation of the periodic states of self-stress at wave-number $\omega=(0,0)$ requires eliminating the modes of rigid-body motion from the row space of the reduced equilibrium matrix, $\widetilde{\mathbf{A}}$. This elimination process is discussed in the following section.

\subsubsection{Elimination of Modes of Rigid-Body Motions from the Row Space of Matrix $\widetilde{\mathbf{A}}$}

The computation of the left-null space of the reduced equilibrium matrix, $\widetilde{\mathbf{A}}$, at wave-number $\omega=(0,0)$, generates a set of independent vectors that represent the modes of mechanisms experienced by the infinite lattice. In a 2D lattice, two of these modes are translational rigid-body motions and the others are internal mechanisms. To determine the states of self-stress that are excited explicitly by the internal mechanisms, the modes of the rigid-body motion have to be eliminated from the row space of the reduced equilibrium matrix; as a result, the states of self-stress are to be re-computed based on the resulting new truncated equilibrium matrix.

We use here the product force vector approach to identify the modes of rigid-body motion from the internal mechanisms (Pellegrino \& Calladine, 1984; 1986). The product force vector approach is a necessary condition to predict the possibility of imparting a positive definite geometrical stiffness. This geometrical stiffness is developed by the inextensional deformation of the infinite structure and the corresponding nodal forces resulting from the triggered states of self-stress. This necessary condition will not be satisfied in the case of rigid-body mechanisms. The following steps explain this process.

First, the null space of the reduced equilibrium matrix, $\widetilde{\mathbf{A}}$, at wave-number $\omega=(0,0)$ is computed to determine the set of reduced states of self-stress which are concatenated into the reduced matrix $\widetilde{\mathbf{S}}$. Applying the procedure of Section 2.1 to the infinite lattice results in the following reduced vector of linear combination constants:

$$
\widetilde{\boldsymbol{\gamma}}=-\left(\varepsilon_{0}\right)\left(\widetilde{\mathbf{S}}^{T} \widetilde{\mathbf{F}} \widetilde{\mathbf{S}}\right)^{-1} \widetilde{\mathbf{S}}^{T} \widetilde{\mathbf{e}}_{l}
$$

where $\widetilde{\mathbf{F}}$ is the reduced diagonal flexibility matrix of the independent elements of the unit cell; $\widetilde{\mathbf{e}}_{l}$ is the reduced vector of element lengths (eqn (14)) applied to the entries corresponding to the redundant independent elements. Using eqn (16), the vector of the reduced generalized states of self-stress, $\widetilde{\mathbf{s}}_{G}$, is given by:

$$
\widetilde{\mathbf{s}}_{G}=\widetilde{\mathbf{S}} \widetilde{\gamma}=-\left(\varepsilon_{0}\right) \widetilde{\mathbf{S}}\left(\widetilde{\mathbf{S}}^{T} \widetilde{\mathbf{F}} \widetilde{\mathbf{S}}\right)^{-1} \widetilde{\mathbf{S}}^{T} \widetilde{\mathbf{e}}_{l}
$$

Second, the product force vector approach is used to check the stiffening effect of the reduced generalized state of self-stress to each mode of mechanisms at wave-number $\omega=(0,0)$. For this purpose we set a nominal strain of $\varepsilon_{0}=|1|$ and we calculate the product force vector for $\varepsilon_{0}=-1$. The negative sign being the shortening of the element which generates a tension field in most lattice topologies. The tension field is necessary to impart positive definite stiffness tensors as the stiffening of the internal mechanisms is developed by the states of self-stress, as explained hereafter. If the stiffening effect is not satisfied at $\varepsilon_{0}=-1$, the computations is repeated for $\varepsilon_{0}=1$. To check the stiffening effect of the reduced generalized state of self-stress for each mode of mechanisms, the following steps are performed.

(i) Expand the reduced modes of mechanisms and global state of self-stress, computed respectively from the left-null space and the null space of the reduced equilibrium matrix. The kinematical displacements of all nodes and the tension force of all elements within the unit cell are then computed as:

$$
\begin{aligned}
\mathbf{D} & =\mathbf{T}_{d} \widetilde{\mathbf{D}} \\
\mathbf{s}_{G} & =\mathbf{T}_{e} \widetilde{\mathbf{s}}_{G}
\end{aligned}
$$

where $\tilde{\mathbf{D}}$ is the reduced mechanisms matrix containing the states of mechanisms of the infinite lattice concatenated into its columns. $\mathbf{T}_{d}$ and $\mathbf{T}_{e}$ are, respectively, the nodal and the element transformation matrices which are used to expand the wave-functions of the nodal and the element deformations from their infinite irreducible representation to the unit cell representation. The transformation matrices are computed using the Bloch's theorem at wave-number $\omega=(0,0)$, as show in (Elsayed, M.S.A., Pasini, D., 2010a; 2010b). 
(ii) Formulate the set of product force vectors corresponding to each individual mechanism by using the expanded modes of mechanisms along with the expanded global state of self-stress obtained in (i). The product force vectors are concatenated into the product force vector matrix, $\mathbf{P}$. The product force vector matrix is then reduced to the irreducible product force vector matrix, $\widetilde{\mathbf{P}}$, of the infinite lattice structure as:

$$
\widetilde{\mathbf{P}}=\mathbf{T}_{d}^{T} \mathbf{P}
$$

(iii) Eliminate the non-pivotal columms in the reduced equilibrium matrix of the infinite lattice to obtain the truncated reduced equilibrium matrix $\widetilde{\mathbf{A}}$.

(iv) Augment the reduced product force vector matrix to the truncated, reduced, equilibrium matrix $\hat{\widetilde{\mathbf{A}}}$ as:

$$
\tilde{\mathbf{A}}_{\text {aug }}=[\hat{\tilde{\mathbf{A}}} \widetilde{\mathbf{P}}]
$$

The reduced row echelon form of the matrix $\left(\tilde{\mathbf{A}}_{\text {aug }}\right)^{T}$ is computed to determine the non-pivotal modes along with their indices. The non-pivotal modes represent the set of mechanisms after applying the stiffening effect of the global state of self-stresses, which - being free - are not affected by the periodic states of self stress. If the number of mechanisms is reduced to two, then these modes are rigid-body motions and the others are internal mechanisms that are stiffened by the global state of self-stress. On the other hand, if this number is greater than two, then an additional filtration step is required to distinguish rigid-body motions from non-stiffened internal mechanisms.

(v) If $g$ is the number of independent nodes within the infinite lattice, then, two modes of rigid-body motion can be defined for the infinite lattice as:

$$
\begin{aligned}
& m_{x}(2(i-1)+1)=1 \\
& m_{x}(2(i-1)+2)=0 \\
& m_{y}(2(i-1)+1)=0 \\
& m_{y}(2(i-1)+2)=1
\end{aligned}
$$

The mechanisms obtained from the augmented equilibrium matrix (eqn (21)) are internal if they are independent from those calculated in eqn (22), else they represent rigid-body motions. Once the indices of rigid-body mechanisms are determined, their corresponding rows are eliminated in the reduced equilibrium matrix to form the matrix $\widetilde{\mathbf{A}}$. The null space of $\widetilde{\mathbf{A}}$ is computed and its states of self-stress are assembled to form the final global state of self-stress, which is in turn expanded (eqn (19)) to generate the tension forces of all elements within the unit cell. These tension forces are used to generate the geometrical stiffness of the lattice structure at wave number $\omega=(0,0)$.

\subsection{Comprehensive Macroscopic Stiffness of Lattice Material}

The stiffness properties of the microscopic infinite lattice structure are homogenized to generate the comprehensive, effective, macroscopic stiffness properties of the lattice material. This is done by using the Cauchy-Born hypothesis (Bhattacharya, 2003; Born \& Huang, 1954; Maugin, 1992) which is used to formulate the Cauchy-Born kinematic boundary condition (Elsayed \& Pasini, 2010a). This boundary condition is an explicit expression of the microscopic nodal deformations in terms of an assumed homogeneous macroscopic strain field applied to the lattice material. The nodal forces are derived by direct substitution of the nodal deformations into the stiffness system of the lattice. The nodal forces and deformations are employed to derive the macroscopic stiffness properties of the pin- and rigid-jointed lattice material using the principle of virtual work (Timoshenko \& Young, 1945) and the Hill-Mandel principle (Maugin, 1992) of macro-homogeneity. Details of this approach can be found in (Elsayed, Pasini, 2010b).

\subsubsection{Cauchy-Born Kinematic Boundary Condition}

Assume $i$ and $j$ are two periodic nodes defined by the position vectors $\mathbf{j}_{l}$ and $\mathbf{j}_{l}+\overrightarrow{\mathbf{R}}$, using the Cauchy-Born hypothesis (Bhattacharya, 2003), the Cauchy-Born kinematic boundary condition can be formulated as: 


$$
\begin{aligned}
& {\left[\begin{array}{c}
d_{i x} \\
d_{i y}
\end{array}\right]=\left[\begin{array}{c}
d_{j x} \\
d_{j y}
\end{array}\right]+\left[\begin{array}{ccc}
\left(x_{i}-x_{j}\right) & 0 & \frac{1}{2}\left(y_{i}-y_{j}\right) \\
0 & \left(y_{i}-y_{j}\right) & \frac{1}{2}\left(x_{i}-x_{j}\right)
\end{array}\right]\left[\begin{array}{c}
\varepsilon_{x x} \\
\varepsilon_{y y} \\
\varepsilon_{x y}
\end{array}\right]} \\
& \text { or }{ }_{p} \mathbf{d}_{i}={ }_{p} \mathbf{d}_{j}+{ }_{p} \mathbf{E} \boldsymbol{\varepsilon} \\
& {\left[\begin{array}{c}
d_{i x} \\
d_{i y} \\
\theta_{i}
\end{array}\right]=\left[\begin{array}{c}
d_{j x} \\
d_{j y} \\
\theta_{j}
\end{array}\right]+\left[\begin{array}{ccc}
\left(x_{i}-x_{j}\right) & 0 & \frac{1}{2}\left(y_{i}-y_{j}\right) \\
0 & \left(y_{i}-y_{j}\right) & \frac{1}{2}\left(x_{i}-x_{j}\right) \\
0 & 0 & 0
\end{array}\right]\left[\begin{array}{c}
\varepsilon_{x x} \\
\varepsilon_{y y} \\
\varepsilon_{x y}
\end{array}\right]} \\
& \text { or }{ }_{r} \mathbf{d}_{i}={ }_{r} \mathbf{d}_{j}+{ }_{r} \mathbf{E} \boldsymbol{\varepsilon}
\end{aligned}
$$

Applying the above boundary condition to the nodal displacement vector, $\mathbf{d}$, of the unit cell results in:

$$
\begin{aligned}
& { }_{p} \mathbf{d}=\left({ }_{p} \mathbf{T}_{d}\right)_{p} \tilde{\mathbf{d}}+{ }_{p} \mathbf{E} \bar{\varepsilon} \\
& { }_{r} \mathbf{d}=\left({ }_{r} \mathbf{T}_{d}\right) \tilde{\mathbf{d}}+{ }_{r} \mathbf{E} \overline{\boldsymbol{\varepsilon}}
\end{aligned}
$$

where ${ }_{p} \mathbf{T}_{d}$ and ${ }_{r} \mathbf{T}_{d}$ are respectively the transformation matrices of the nodal degrees of freedom for pin- and rigid-jointed unit cells. Eqns (24) are the pin- and the rigid-jointed kinematic boundary conditions of the Cauchy-Born hypothesis.

Substituting eqns (24) into the stiffness system of the unit cell, eqns (3), results in:

$$
\begin{aligned}
& { }_{p} \mathbf{K}_{C}\left\{\left({ }_{p} \mathbf{T}_{d}\right)_{p} \widetilde{\mathbf{d}}+{ }_{p} \mathbf{E}-\overline{\boldsymbol{\varepsilon}}\right\}=\left\{{ }_{p} \mathbf{K}_{E}^{\text {bar }}+{ }_{p} \mathbf{K}_{G}^{\text {bar }}\right)\left\{\left({ }_{p} \mathbf{T}_{d}\right)_{p} \widetilde{\mathbf{d}}+{ }_{p} \mathbf{E} \boldsymbol{\varepsilon}\right\}=\mathbf{f} \\
& { }_{r} \mathbf{K}_{C}\left\{\left({ }_{r} \mathbf{T}_{d}\right)_{r} \widetilde{\mathbf{d}}+{ }_{r} \mathbf{E} \mathbf{\varepsilon}\right\}=\left\{{ }_{r} \mathbf{K}_{E}^{\text {bar }}+{ }_{r} \mathbf{K}_{E}^{\text {beam }}+{ }_{r} \mathbf{K}_{G}^{\text {frame }}\right\}\left\{\left({ }_{r} \mathbf{T}_{d}\right)_{r} \widetilde{\mathbf{d}}+{ }_{r} \mathbf{E} \mathbf{\varepsilon}\right\}=\mathbf{f}
\end{aligned}
$$

Multiplying both sides of eqns (25) by the transpose of the transformation matrices, ${ }_{p} \mathbf{T}_{d}$ and ${ }_{r} \mathbf{T}_{d}$, respectively, and applying the anti-periodic constraints necessary for the static equilibrium of the lattice, gives:

$$
\begin{aligned}
& \left({ }_{p} \mathbf{T}_{d}\right)^{T}\left\{{ }_{p} \mathbf{K}_{E}^{\text {bar }}+{ }_{p} \mathbf{K}_{G}^{\text {bar }}\right)_{p}\left(\mathbf{T}_{d}\right)_{p} \widetilde{\mathbf{d}}=-\left({ }_{p} \mathbf{T}_{d}\right)^{T}\left\{{ }_{p} \mathbf{K}_{E}^{\text {bar }}+{ }_{p} \mathbf{K}_{G}^{\text {bar }}\right\}_{p} \mathbf{E} \boldsymbol{\overline { \varepsilon }} \\
& \left.\left({ }_{r} \mathbf{T}_{d}\right)^{T}\left\{{ }_{r} \mathbf{K}_{E}^{\text {bar }}+{ }_{r} \mathbf{K}_{E}^{\text {beam }}+{ }_{r} \mathbf{K}_{G}^{\text {frame }}\right\}_{r} \mathbf{T}_{d}\right)_{r} \widetilde{\mathbf{d}}=-\left({ }_{r} \mathbf{T}_{d}\right)^{T}\left\{{ }_{r} \mathbf{K}_{E}^{\text {bar }}+{ }_{r} \mathbf{K}_{E}^{\text {beam }}+{ }_{r} \mathbf{K}_{G}^{\text {frame }}\right\}_{r} \mathbf{E} \overline{\boldsymbol{\varepsilon}}
\end{aligned}
$$

The first equation in (26) can be split into two matrix systems as:

$$
\begin{aligned}
& \left({ }_{p} \widetilde{\mathbf{K}}_{E}^{\text {bar }}\right)_{p} \widetilde{\mathbf{d}}=-\left({ }_{p} \mathbf{T}_{d}\right)^{T}\left({ }_{p} \mathbf{K}_{E}^{b a r}\right)_{p} \mathbf{E} \boldsymbol{\varepsilon} \\
& \left({ }_{p} \widetilde{\mathbf{K}}_{G}^{b a r}\right)_{p} \widetilde{\mathbf{d}}=-\left({ }_{p} \mathbf{T}_{d}\right)^{T}\left({ }_{p} \mathbf{K}_{G}^{b a r}\right)_{p} \mathbf{E} \overline{\boldsymbol{\varepsilon}}
\end{aligned}
$$

where ${ }_{p} \widetilde{\mathbf{K}}_{E}^{\text {bar }}=\left({ }_{p} \mathbf{T}_{d}\right)^{T}\left({ }_{p} \mathbf{K}_{E}^{\text {bar }}\right)_{p} \mathbf{T}_{d}$ and ${ }_{p} \widetilde{\mathbf{K}}_{G}^{\text {bar }}=\left({ }_{p} \mathbf{T}_{d}\right)^{T}{ }_{p}\left(\mathbf{K}_{G}^{\text {bar }}\right)_{p} \mathbf{T}_{d}$ are, respectively, the material and the geometrical stiffness matrices of the infinite periodic structure with pin joints.

Similarly, the second eqn in (26) can be split into three matrix systems as: 


$$
\begin{aligned}
& \left({ }_{r} \widetilde{\mathbf{K}}_{E}^{\text {bar }}\right)_{r} \widetilde{\mathbf{d}}=-\left({ }_{r} \mathbf{T}_{d}\right)^{T}\left({ }_{r} \mathbf{K}_{E}^{\text {bar }}\right)_{r} \mathbf{E} \overline{\boldsymbol{\varepsilon}} \\
& \left({ }_{r} \widetilde{\mathbf{K}}_{E}^{\text {beam }}\right)_{r} \widetilde{\mathbf{d}}=-\left({ }_{r} \mathbf{T}_{d}\right)^{T}\left({ }_{r} \mathbf{K}_{E}^{\text {beam }}\right)_{r} \mathbf{E} \overline{\boldsymbol{\varepsilon}} \\
& \left({ }_{r} \widetilde{\mathbf{K}}_{G}^{\text {frame }}\right)_{r} \widetilde{\mathbf{d}}=-\left({ }_{r} \mathbf{T}_{d}\right)^{T}\left({ }_{r} \mathbf{K}_{G}^{\text {frame }}\right)_{r} \mathbf{E} \boldsymbol{\varepsilon}
\end{aligned}
$$

where ${ }_{r} \widetilde{\mathbf{K}}_{E}^{\text {bar }}=\left({ }_{r} \mathbf{T}_{d}\right)^{T}\left({ }_{r} \mathbf{K}_{E}^{\text {bar }}\right)_{r} \mathbf{T}_{d},{ }_{r} \widetilde{\mathbf{K}}_{E}^{\text {beam }}=\left({ }_{r} \mathbf{T}_{d}\right)^{T}\left({ }_{r} \mathbf{K}_{E}^{\text {beam }}\right)_{r} \mathbf{T}_{d} \quad$ and ${ }_{r} \widetilde{\mathbf{K}}_{G}^{\text {frame }}=\left({ }_{r} \mathbf{T}_{d}\right)^{T}\left({ }_{r} \mathbf{K}_{G}^{\text {frame }}\right)_{r} \mathbf{T}_{d}$ are, respectively, the material axial, the material bending and the geometrical stiffness matrices of the infinite periodic structure with rigid joints.

\subsubsection{Nodal Deformations in Terms of Macroscopic Strain Field}

To obtain the nodal deformations in terms of a macroscopic strain field, both sides of eqns (27) should be multiplied by the inverse of the reduced stiffness matrices of the infinite lattice structure. To invert these matrices, we need first to remove all the modes of both rigid-body motions and internal mechanisms, which would make the matrices singular. Once the modes are eliminated, the resulting stiffness matrices (subscript "red") can be inverted to obtain the following expressions of nodal displacements:

$$
\begin{aligned}
& { }_{p} \widetilde{\mathbf{d}}_{E}^{\text {bar }}=-\left({ }_{p} \widetilde{\mathbf{K}}_{E}^{\text {bar }}\right)_{\text {red }}^{-1}\left({ }_{p} \mathbf{T}_{d}\right)^{T}\left({ }_{p} \mathbf{K}_{E}^{\text {bar }}\right)_{p} \mathbf{E} \overline{\boldsymbol{\varepsilon}} \\
& { }_{p} \tilde{\mathbf{d}}_{G}^{\text {bar }}=-\left({ }_{p} \widetilde{\mathbf{K}}_{G}^{\text {bar }}\right)_{\text {red }}^{-1}\left({ }_{p} \mathbf{T}_{d}\right)^{T}\left({ }_{p} \mathbf{K}_{G}^{b a r}\right)_{p} \mathbf{E} \overline{\boldsymbol{\varepsilon}} \\
& { }_{r} \widetilde{\mathbf{d}}_{E}^{b a r}=-\left({ }_{r} \widetilde{\mathbf{K}}_{E}^{b a r}\right)_{r e d}^{-1}\left({ }_{r} \mathbf{T}_{d}\right)^{T}\left({ }_{r} \mathbf{K}_{E}^{b a r}\right)_{r} \mathbf{E} \overline{\boldsymbol{\varepsilon}} \\
& { }_{r} \tilde{\mathbf{d}}_{E}^{\text {beam }}=-\left({ }_{r} \widetilde{\mathbf{K}}_{E}^{\text {beam }}\right)_{\text {red }}^{-1}\left({ }_{r} \mathbf{T}_{d}\right)^{T}\left({ }_{r} \mathbf{K}_{E}^{\text {beam }}\right)_{r} \mathbf{E} \overline{\boldsymbol{\varepsilon}} \\
& { }_{r} \tilde{\mathbf{d}}_{G}^{\text {frame }}=-\left({ }_{r} \widetilde{\mathbf{K}}_{G}^{\text {frame }}\right)_{\text {red }}^{-1}\left({ }_{r} \mathbf{T}_{d}\right)^{T}\left({ }_{r} \mathbf{K}_{G}^{\text {frame }}\right)_{r} \mathbf{E} \overline{\boldsymbol{\varepsilon}}
\end{aligned}
$$

Where ${ }_{p} \tilde{\mathbf{d}}_{E}^{b a r}$ and ${ }_{p} \tilde{\mathbf{d}}_{G}^{\text {bar }}$ are, respectively, the nodal deformations corresponding to the material and the geometrical stiffness resistances of the pin-jointed lattice. On the other hand, ${ }_{r} \tilde{\mathbf{d}}_{E}^{\text {bar }},{ }_{r} \tilde{\mathbf{d}}_{E}^{\text {beam }} \underset{r}{{ }_{r}}$ and $\mathbf{d}_{G}$ are, respectively, the nodal deformations corresponding to the material axial, the material bending and the geometrical stiffness of the rigid-jointed lattices.

The generalized deformation of all nodes within the unit cell can be computed by substituting eqns (28) into eqns (24). For the expressions of the generalized microscopic nodal forces, we substitute the generalized nodal deformation vectors into the stiffness system of the unit cell (eqns (3)) for pin- and rigid-jointed lattices. The generalized nodal deformations and forces are given by:

$$
\begin{gathered}
\mathbf{d}_{E}^{\text {bar }}={ }_{p}^{d} \mathbf{M}_{E}^{\text {bar }} \overline{\boldsymbol{\varepsilon}},{ }_{p} \mathbf{f}_{E}^{\text {bar }}={ }_{p}^{f} \mathbf{M}_{E}^{\text {bar }} \overline{\boldsymbol{\varepsilon}} \\
\mathbf{d}_{G}^{\text {bar }}={ }_{p}^{d} \mathbf{M}_{G}^{\text {bar }} \overline{\boldsymbol{\varepsilon}},{ }_{p} \mathbf{f}_{G}^{\text {bar }}={ }_{p}^{f} \mathbf{M}_{G}^{\text {bar }} \overline{\boldsymbol{\varepsilon}}, \\
{ }_{r} \mathbf{d}_{E}^{\text {bar }}={ }_{r}^{d} \mathbf{M}_{E}^{\text {bar }} \overline{\overline{\boldsymbol{\varepsilon}}}, \quad{ }_{r} \mathbf{f}_{E}^{\text {bar }}={ }_{r}^{f} \mathbf{M}_{E}^{\text {bar }} \overline{\boldsymbol{\varepsilon}} \\
{ }_{r} \mathbf{d}_{E}^{\text {beam }}={ }_{r}^{d} \mathbf{M}_{E}^{\text {beam }} \overline{\overline{\boldsymbol{\varepsilon}},},{ }_{r} \mathbf{f}_{E}^{\text {beam }}={ }_{r}^{f} \mathbf{M}_{E}^{\text {beam }} \overline{\overline{\boldsymbol{\varepsilon}}}, \\
{ }_{r} \mathbf{d}_{G}^{\text {frame }}={ }_{r}^{d} \mathbf{M}_{G}^{\text {frame }} \overline{\overline{\boldsymbol{\varepsilon}},}{ }_{r} \mathbf{f}_{G}^{\text {frame }}={ }_{r}^{f} \mathbf{M}_{G}^{\text {frame }} \overline{\overline{\boldsymbol{\varepsilon}}}
\end{gathered}
$$

\subsubsection{Homogenized Macroscopic Stiffness of Lattice Material}

From expressions (29) and through the principle of virtual work (Timoshenko and Young, 1945) and the Hill-Mandel principle of macro-homogeneity (Maugin, 1992), we calculate the comprehensive macroscopic 
stiffness properties of pin- and rigid-jointed lattice materials for a 2D lattice with out-of-plane thickness of unity as:

$$
\begin{aligned}
& \mathbf{K}_{L E}^{\text {bar }}=\left(\frac{E}{2|\mathrm{Y}|}\right)\left(\frac{\rho_{L}}{c_{T}^{\rho}}\right)\left(\left({ }_{p}^{f} \mathbf{M}_{E}^{\text {bar }}\right)^{T}\left({ }_{p}^{d} \mathbf{M}_{E}^{\text {bar }}\right)\right) \\
& { }_{p} \mathbf{K}_{L G}^{\text {bar }}=\left(\frac{E}{2|\mathrm{Y}|}\right)\left(\frac{\rho_{L}}{c_{T}^{\rho}}\right)\left(\left({ }_{p}^{f} \mathbf{M}_{G}^{\text {bar }}\right)^{T}\left({ }_{p}^{d} \mathbf{M}_{G}^{\text {bar }}\right)\right) \\
& { }_{r} \mathbf{K}_{L E}^{\text {bar }}=\left(\frac{E}{2|\mathrm{Y}|}\right)\left(\frac{\rho_{L}}{c_{T}^{\rho}}\right)\left(\left({ }_{r}^{f} \mathbf{M}_{E}^{\text {bar }}\right)^{T}\left({ }_{r}^{d} \mathbf{M}_{E}^{\text {bar }}\right)\right) \\
& { }_{r} \mathbf{K}_{L E}^{\text {beam }}=\left(\frac{E}{2|\mathrm{Y}|}\right)\left(\frac{\rho_{-}}{\rho_{L}}\right)\left(\left({ }_{r}^{f} \mathbf{M}_{E}^{\text {beam }}\right)^{T}\left({ }_{r}^{d} \mathbf{M}_{E}^{\text {beam }}\right)\right) \\
& { }_{r} \mathbf{K}_{L G}^{\text {frame }}=\left(\frac{E}{2|\mathrm{Y}|}\right)\left(\frac{{ }^{-}}{\rho_{L}}\right)\left(\left({ }_{r}^{f} \mathbf{M}_{G}^{\text {frame }}\right)^{T}\left({ }_{r}^{d} \mathbf{M}_{G}^{\text {frame }}\right)\right)
\end{aligned}
$$

where $c_{T}^{\rho}$ and $|\mathrm{Y}|$ are, respectively, a density constant that depends on the topology of the unit cell and the in-plane area of the unit cell enclosed within the cell envelope. We recall the expression of the relative density for a 2D lattice material:

$$
\bar{\rho}_{L}=\frac{\rho_{L}}{\rho}=c_{T}^{\rho}\left(\frac{H}{L}\right)
$$

where $H$ is a unified thickness of the microscopic cell element in the plane of the 2D lattice. $\rho_{L}, \rho_{L}$ and $\rho$ are respectively, the lattice material relative density, the lattice material density and the density of the solid material.

From eqns (31), we write the comprehensive stiffness of a lattice material as:

$$
\begin{gathered}
{ }_{p} \mathbf{K}_{L C}={ }_{p} \mathbf{K}_{L E}^{\text {bar }}+{ }_{p} \mathbf{K}_{L G}^{\text {bar }} \\
{ }_{r} \mathbf{K}_{L C}={ }_{r} \mathbf{K}_{L E}^{\text {bar }}+{ }_{r} \mathbf{K}_{L E}^{\text {beam }}+{ }_{r} \mathbf{K}_{L G}^{\text {frame }}
\end{gathered}
$$

where ${ }_{p} \mathbf{K}_{L E}^{b a r}={ }_{r} \mathbf{K}_{L E}^{b a r}$. Once the macroscopic stiffness matrix is computed, the macroscopic compliance matrix can be obtained along with the corresponding material elastic moduli.

\section{Example}

To illustrate the steps of the methodology presented in this paper, we select the 2D Kagome as a paradigm topology (Figure (1)). To obtain the comprehensive stiffness, we start from the reduced equilibrium matrix of the infinite Kagome lattice at wave-number $\omega=(0,0)$ :

$$
\tilde{\mathbf{A}}=\left[\begin{array}{cccccc}
-0.5 & -0.866 & 0.5 & 0.866 & 0 & 0 \\
0 & 0 & -1 & 0 & 1 & 0 \\
0.5 & -0.866 & 0 & 0 & -0.5 & 0.866 \\
0.5 & 0.866 & -0.5 & -0.866 & 0 & 0 \\
0 & 0 & 1 & 0 & -1 & 0 \\
-0.5 & 0.866 & 0 & 0 & 0.5 & -0.866
\end{array}\right]
$$


The null space of the reduced equilibrium matrix, eqn (33), is calculated to determine the states of self-stress matrix, $\widetilde{\mathbf{S}}$; the resulting modes are concatenated into the columns of the matrix $\widetilde{\mathbf{S}}$. From the computation of the reduced row echelon form of (33), we observe that the indices 4,5 and 6 in the column space are non-pivotal modes. Hence, we can eliminate them from the reduced equilibrium matrix, $\mathbf{A}$, to obtain the reduced truncated equilibrium matrix, $\widetilde{\mathbf{A}}$.

To determine the states of mechanisms of the Kagome lattice, the left-null space of the equilibrium matrix in eqn (33) is computed and the resulting modes are concatenated into the columns of matrix $\mathbf{D}$, where $\mathbf{D}$ is the reduced kinematic matrix. Three mechanisms are recovered from this computation, two out of the three modes are rigid-body motions. To determine accurately the states of self-stress associated with internal mechanisms at wave-number $\omega=(0,0)$, we eliminate the modes of rigid-body motions from the row space of the matrix $\widetilde{\mathbf{A}}$. To distinguish modes of rigid-body motions from internal mechanisms, we resort to the product force vector analysis.

The reduced flexibility matrix, $\widetilde{\mathbf{F}}$, and the reduced vector of imposed elongations, $\widetilde{\mathbf{e}}_{0}$, are computed as:

$$
\widetilde{\mathbf{F}}=\frac{L}{E A}\left[\begin{array}{cccccc}
1 & 0 & 0 & 0 & 0 & 0 \\
0 & 1 & 0 & 0 & 0 & 0 \\
0 & 0 & 1 & 0 & 0 & 0 \\
0 & 0 & 0 & 1 & 0 & 0 \\
0 & 0 & 0 & 0 & 1 & 0 \\
0 & 0 & 0 & 0 & 0 & 1
\end{array}\right], \quad \widetilde{\mathbf{e}}_{0}=\varepsilon_{0}\left[\begin{array}{l}
0 \\
0 \\
0 \\
1 \\
1 \\
1
\end{array}\right]
$$

Substituting eqn (34) into eqn (12) yields the reduced vector of linear combination constants of the Kagome lattice as:

$$
\widetilde{\gamma}=\left(\varepsilon_{0}\right)\left[\begin{array}{lll}
-0.5 & -0.5 & -0.5
\end{array}\right]^{T}
$$

Substituting eqn (35) into eqn (17), results in the reduced global state of self-stress of the Kagome lattice at wave-number, $\omega=(0,0)$ as:

$$
\widetilde{\mathbf{s}}_{G}=\left(\varepsilon_{0}\right)\left[\begin{array}{llllll}
-0.5 & -0.5 & -0.5 & -0.5 & -0.5 & -0.5
\end{array}\right]^{T}
$$

If we impose a nominal imposed strain $\varepsilon_{0}=-1$, we obtain the global state of self-stress and the global mechanisms which are used to formulate the product force vector matrix, $\mathbf{P}$. This matrix is then reduced to form the reduced product force vector matrix, $\widetilde{\mathbf{P}}$. Augmenting the reduced product force vector matrix, with the truncated reduced equilibrium matrix gives the expanded reduced equilibrium matrix as:

$$
\widetilde{\mathbf{A}}_{\text {aug }}=\left[\begin{array}{ccc||ccc}
-0.5 & 0 & 0.5 & 1.732 & 0 & -1.732 \\
-0.866 & 0 & -0.866 & 0 & 0 & 0 \\
0.5 & -1 & 0 & -0.866 & 0 & 0.866 \\
0.866 & 0 & 0 & 1.5 & 0 & -1.5 \\
0 & 1 & -0.5 & -0.866 & 0 & 0.866 \\
0 & 0 & 0.866 & -1.5 & 0 & 1.5
\end{array}\right]
$$

To determine the modes of the un-stiffened mechanisms along with their indices, we calculate the left-null space of the augmented reduced equilibrium matrix (eqn (35)) of the Kagome lattice as:

$$
\tilde{\mathbf{D}}=\left[\begin{array}{llllll}
1 & 0 & 1 & 0 & 1 & 0 \\
0 & 1 & 0 & 1 & 0 & 1
\end{array}\right]^{T}
$$

The first and the second columns of the kinematic matrix, $\tilde{\mathbf{D}}$, (eqn (36)) of the Kagome lattice, represent translational rigid-body motions in the $x$ and the $y$ directions, respectively. Since for the Kagome lattice there are only two mechanisms, which both represent rigid-body motions, no additional filtration is required. The indices of the two mechanisms are found to be the $5^{\text {th }}$ and $6^{\text {th }}$ indices in the row space of the augmented reduced 
equilibrium matrix. The nodal degrees of freedom corresponding to these modes are then removed from the reduced equilibrium matrix to obtain the matrix $\widetilde{\mathbf{A}}$. To determine the final sets of states of self-stress and states of internal mechanisms, the null space and the left-null space of the equilibrium matrix $\widetilde{\mathbf{A}}$ are computed, respectively, as:

$$
\tilde{\mathbf{D}}=\left[\begin{array}{llll}
0.866 & 0.5 & 1 & 1
\end{array}\right]^{T}, \quad \widetilde{\mathbf{S}}=\left[\begin{array}{llllll}
1 & 0 & 0 & 1 & 0 & 0 \\
0 & 1 & 0 & 0 & 1 & 0 \\
0 & 0 & 1 & 0 & 0 & 1
\end{array}\right]^{T}
$$

The results above allow writing the vector of linear combination constants which is then used to compute the final global state of self-stress. For the Kagome lattice, this results in:

$$
\widetilde{\gamma}=\left(\varepsilon_{0}\right)\left[\begin{array}{lll}
-0.5 & -0.5 & -0.5
\end{array}\right]^{T}, \quad \widetilde{\mathbf{s}}_{G}=\left(\varepsilon_{0}\right)\left[\begin{array}{llllll}
-0.5 & -0.5 & -0.5 & -0.5 & -0.5 & -0.5
\end{array}\right]^{T}
$$

Eqn (19) is used to expand the final global state of self-stress and formulate the geometrical stiffness of the unit cell structure. After homogenization, the geometrical and material stiffness are combined to obtain the comprehensive stiffness of the pin- and the rigid-jointed Kagome lattice materials as reported below.

For a pin-jointed Kagome lattice material:

$$
{ }_{p} \overline{\mathbf{K}}_{L C}=\frac{{ }_{p} \mathbf{K}_{L C}}{E}=\bar{\rho}_{L}\left[\begin{array}{ccc}
0.375 & 0.125 & 0 \\
0.125 & 0.375 & 0 \\
0 & 0 & 0.125
\end{array}\right]+\left(\varepsilon_{0}\right)\left(-\rho_{L}\right)\left[\begin{array}{ccc}
-0.063 & 0.063 & 0 \\
0.063 & -0.063 & 0 \\
0 & 0 & -0.063
\end{array}\right]
$$

For a rigid-jointed Kagome lattice material:

$$
{ }_{r} \overline{\mathbf{K}}_{L C}=\frac{{ }_{r} \mathbf{K}_{L C}}{E}=\rho_{L}\left[\begin{array}{ccc}
0.375 & 0.125 & 0 \\
0.125 & 0.375 & 0 \\
0 & 0 & 0.125
\end{array}\right]+{ }^{3}\left[\begin{array}{ccc}
0.021 & -0.021 & 0 \\
-0.021 & 0.021 & 0 \\
0 & 0 & 0.021
\end{array}\right]+\left(\varepsilon_{0}\right)\left(-\rho_{L}\right)\left[\begin{array}{ccc}
-0.038 & 0.038 & 0 \\
0.038 & -0.038 & 0 \\
0 & 0 & -0.038
\end{array}\right]
$$

where $\bar{\rho}_{L}=1.732\left(\frac{H}{L}\right)$.

The elastic moduli and Poisson's ratios of the pin- and rigid-jointed Kagome lattice, based on comprehensive stiffness analysis, are given by:

For a pin-jointed Kagome lattice

$$
\left({ }_{p} \bar{E}_{L}\right)_{x x}=\left({ }_{p} \bar{E}_{L}\right)_{y y}=\bar{\rho}_{L} \frac{\left(\varepsilon_{0}-2\right)}{\left(\varepsilon_{0}-6\right)},{ }_{p} \bar{G}_{L}=\frac{-1}{16} \bar{\rho}_{L}\left(\varepsilon_{0}-2\right),\left({ }_{p} v_{L}\right)_{x y}=\left({ }_{p} v_{L}\right)_{y x}=-\frac{\left(\varepsilon_{0}+2\right)}{\left(\varepsilon_{0}-6\right)}
$$

For a rigid-jointed Kagome lattice

$$
\begin{aligned}
\left({ }_{r} \bar{E}_{L}\right)_{x x} & =\left({ }_{r} \bar{E}_{L}\right)_{y y}=\bar{\rho}_{L} \frac{\left(1250+208 \bar{\rho}_{L}^{2}-375 \varepsilon_{0}\right)}{3750+208 \overline{\rho_{L}-375 \varepsilon_{0}}},{ }_{r} \bar{G}_{L}=\frac{\bar{\rho}_{L}}{10^{4}}\left(1250+208 \bar{\rho}_{L}-375 \varepsilon_{0}\right), \\
\left({ }_{r} v_{L}\right)_{x y} & =\left({ }_{r} v_{L}\right)_{y x}=-\frac{\left(-1250+208 \bar{\rho}_{L}-375 \varepsilon_{0}\right)}{3750+208 \bar{\rho}_{L}-375 \varepsilon_{0}}
\end{aligned}
$$

\section{The Contribution of the Geometrical Stiffness to the Stiffness Resistance of Lattice Materials}

In this section, the elastic properties of the Kagome and the $3^{3} \cdot 4^{2}$ lattices are examined to discuss the contribution of their geometrical stiffness and impact on the stiffness properties of the material. 


\subsection{The Kagome Lattice Material}

In Figure (2), the comprehensive stiffness properties of the Kagome lattice material is plotted for a relative density $\rho_{L}=0.1$, and a nominal strain range $\varepsilon_{0}=0:-0.2$. The figure shows the variation of the elastic moduli of the Kagome lattice material against the nominal strain of the solid material. A minor contribution of the geometrical stiffness to the comprehensive stiffness can be observed for the Kagome lattice. The reason for this is that the Kagome lattice is stretching dominated and thus the contribution of the material stiffness dominates the other terms.

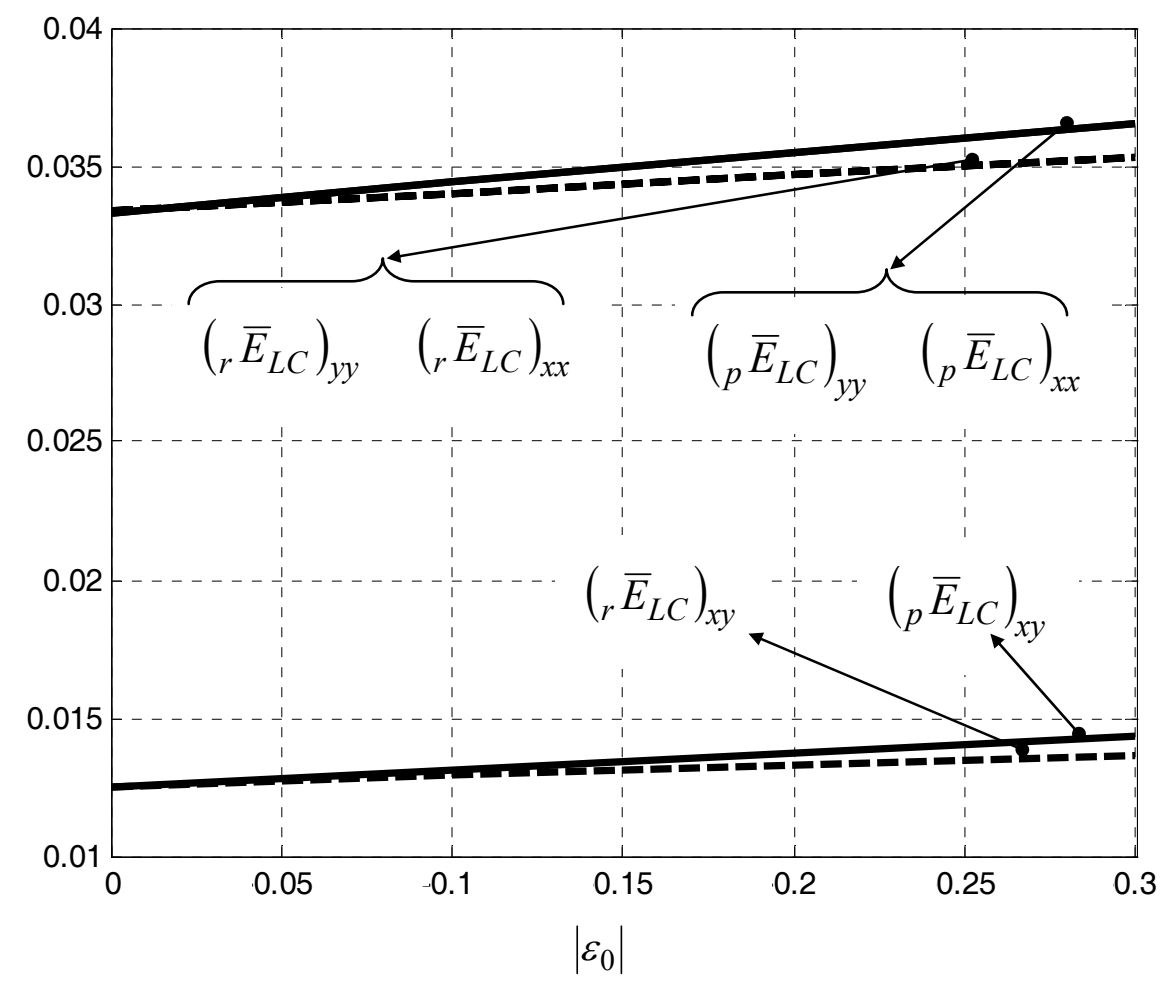

Figure 2. Variation of the elastic moduli of the Kagome lattice with an applied nominal strain

\subsection{The $3^{3} 4^{2}$ Lattice Material}

Plotted for a relative density of $\overline{\rho_{L}}=0.1$, Figure (3) shows the variation of the lattice shear moduli versus an applied nominal strain in the range $\varepsilon_{0}=0:-0.2$. The curves show that the geometrical stiffness of the pin-jointed $3^{3} \cdot 4^{2}$ lattice material contributes significantly to its comprehensive stiffness. In particular, Figure (4) shows that the geometrical stiffness can strongly enhance the stiffness resistance of a lattice topology, e.g. $3^{3} \cdot 4^{2}$ lattice, while have a minor effect on others, e.g. the Kagome lattice. The Young's moduli in both the in-plane directions of the former are higher than those of the latter for the whole range of nominal strain under investigation. The rank of the shear modulus, however, changes in a range of nominal strain, as defined by the value $\varepsilon_{0}=-0.234$. When no geometrical stiffness is taken into account, the Kagome lattice material is better and its shear modulus outperforms up to $\varepsilon_{0}<-0.234$. For a nominal strain above this threshold, however, the shear resistance of the $3^{3} \cdot 4^{2}$ lattice prevails.

The above demonstrates that the stiffness behaviour of a given lattice topology can be theoretically boosted if a nominal strain is applied to one of the cell member. Further work is required to verify experimentally the results found in this paper. Our plan is to replace one or more redundant microelements of the lattice with a smart material, which upon excitation can trigger the geometrical stiffness of the lattice.

\section{Conclusion}

The paper presented a matrix-based approach to derive the comprehensive stiffness of infinite periodic frameworks and lattice materials. The methodology can be applied to pin- and rigid-jointed structures and periodic cellular solids. Applied in detail to the Kagome lattice, the procedure has enabled to quantify the impact of the terms describing the geometrical stiffness onto the comprehensive stiffness. The homogenized 
comprehensive stiffness of 12 lattice materials with pin- and rigid-joints has been characterized and given in the Appendix.

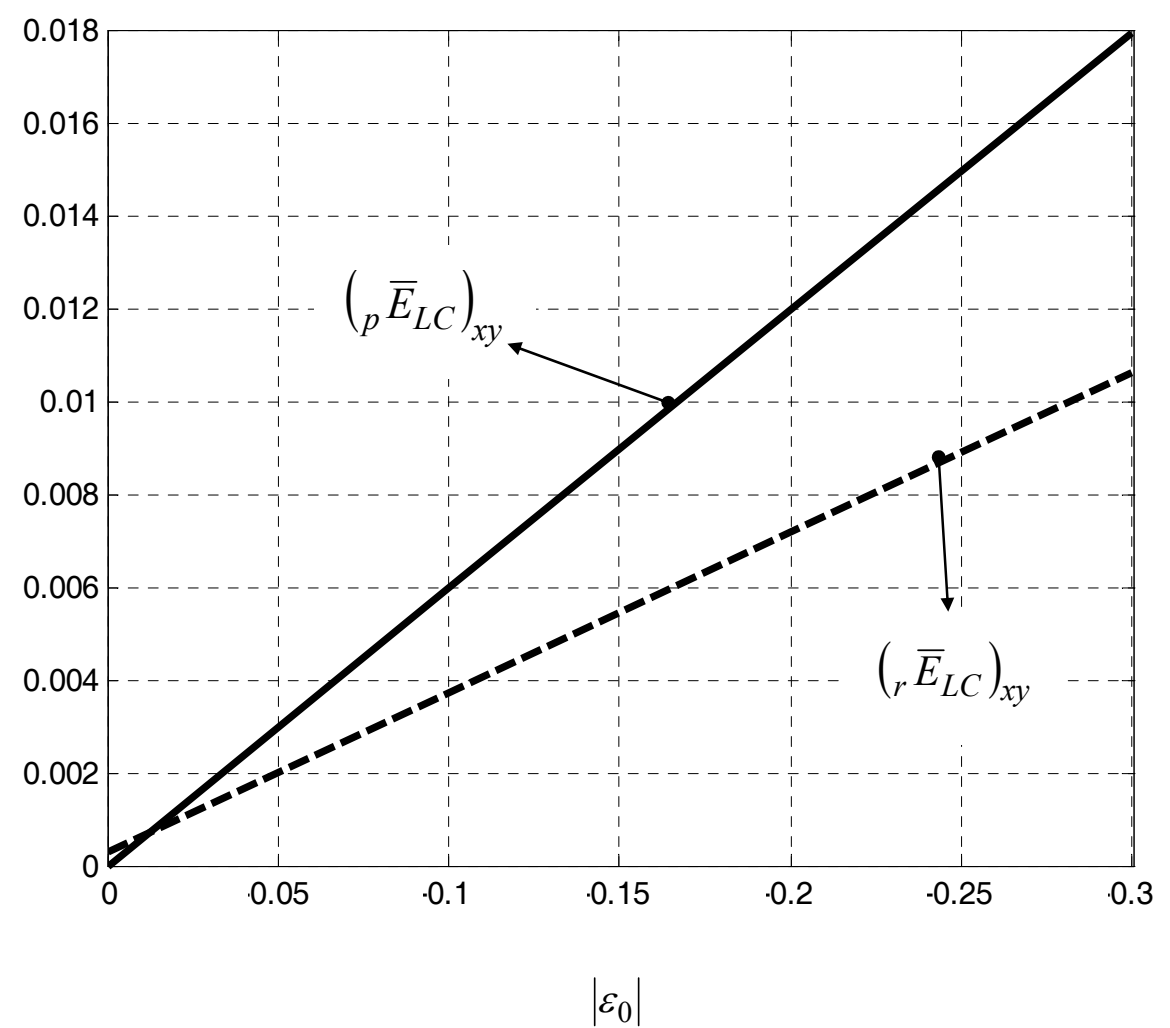

Figure 3. Variation of the shear moduli of the $3^{3} \cdot 4^{2}$ lattice material with an applied nominal strain

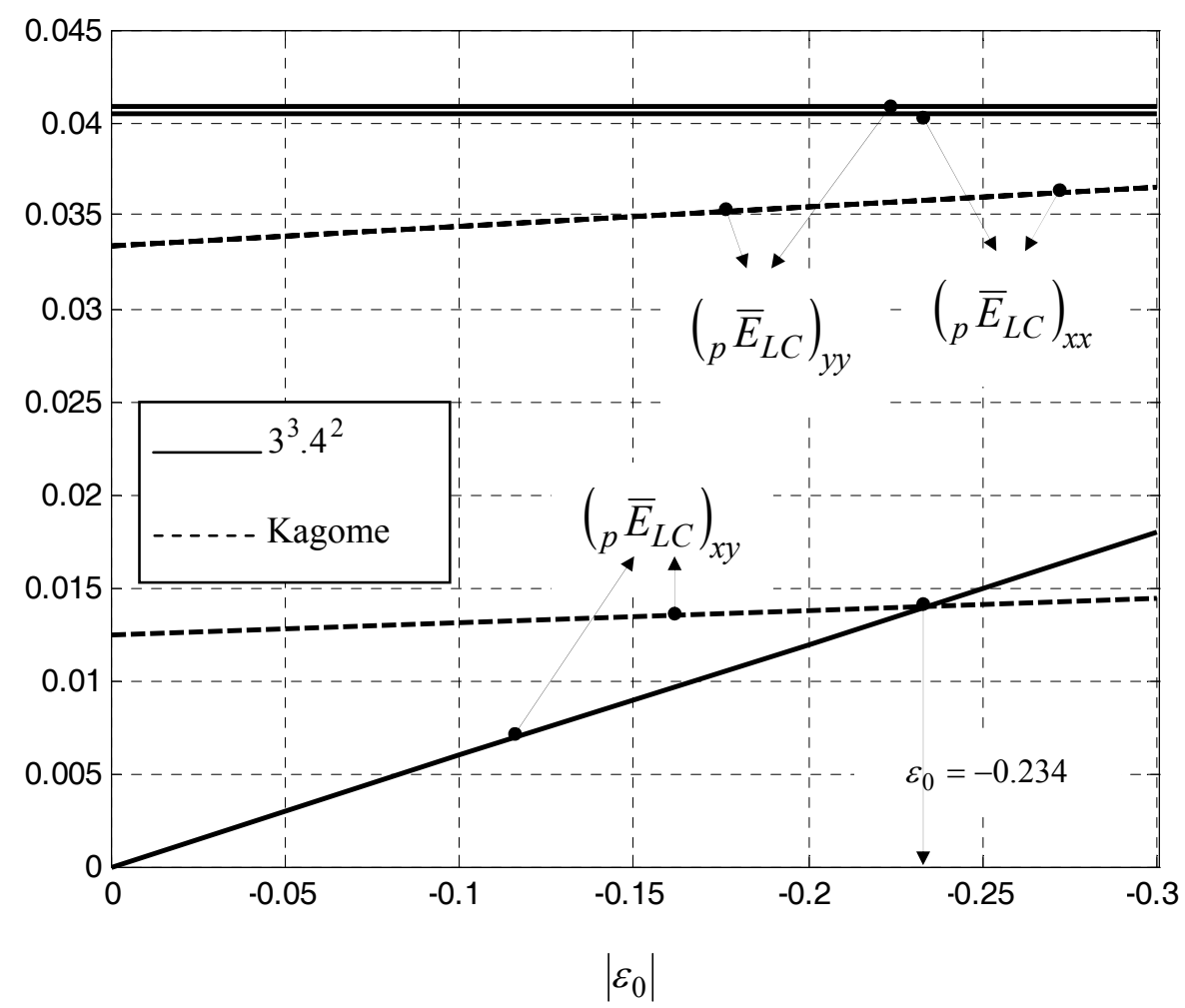

Figure 4. Comparison of the elastic moduli of the $3^{3} \cdot 4^{2}$ lattice and the Kagome lattice 


\section{References}

Bhattacharya, K. (2003). Microstructure of Martensite: Why it Forms and How it Gives Rise to the Shape-Memory Effect. Oxford series on materials modeling, 2. New York: Oxford University Press.

Bickford, W. B. (1998). Advanced mechanics of materials. Menlo Park: Addison- Wesley.

Born, M., \& Huang, K. (1954). Dynamical Theory of Crystal Lattices. Oxford: Clarendon Press.

Brillouin, L. (1946). Wave propagation in periodic structures: electric filters and crystal lattices. International series in pure and applied physics. New York: McGraw- Hill.

Calladine, C. R. (1978). Buckminster Fuller's "Tensegrity" structures and Clerk Maxwell's rules for the construction of stiff frames. International Journal of Solids and Structures, 14, 161-172. http://dx.doi.org/10.1016/0020-7683(78)90052-5

Calladine, C. R. (1982). Modal stiffness of a pre-tensioned cable net. International Journal of Solids and Structures, 18, 829-846. http://dx.doi.org/10.1016/0020-7683(82)90068-3

Calladine, C. R., \& Pellegrino, S. (1991). First-order infinitesimal mechanisms. International Journal of Solids and Structures, 27, 505-515. http://dx.doi.org/10.1016/0020-7683(91)90137-5

Connelly, R., \& Whiteley, W. (1996). Second-order rigidity and prestress stability for tensegrity frameworks. SIAM Journal on Discrete Mathematics, 9(3), 453-491. http://dx.doi.org/10.1137/S0895480192229236

Cook, R. D., Malkus, D. S., Plesha, M. E., \& Witt, R. J. (2001). Concepts and Applications of Finite Element Analysis (4th ed). Wiley.

Deshpande, V. S., Ashby, M. F., \& Fleck, N. A. (2001a). Foam topology bending versus stretching dominated architectures. Acta Materialia, 49(6), 1035-1040. http://dx.doi.org/10.1016/S1359-6454(00)00379-7

Elsayed, M. S. A., \& Pasini, D. (2010a). Analysis of the Elastostatic Specific Stiffness of 2D Stretching-Dominated Lattice Materials. Journal of Mechanics of Materials, 42(7), 709-725. http://dx.doi.org/10.1016/j.mechmat.2010.05.003

Elsayed, M. S. A., \& Pasini, D. (2011b). Stiffness Properties of Periodic Cellular Solids with Rigid Joints. Submitted to the International Journal of Solids and Structures, Manuscript Number: IJSS 10-777.

Guest, S. D., \& Hutchinson, J. W. (2003). On the Determinacy of Repetitive Structures. J. of Mechanics and Physics of Solids, 51(3), 383-391. http://dx.doi.org/10.1016/S0022-5096(02)00107-2

Guest, S. (2006). The Stiffness of Prestressed frameworks: A unifying approach. International Journal of Solids and Structures, 43, 842-854. http://dx.doi.org/10.1016/j.ijsolstr.2005.03.008

Hill, R. (1963). Elastic Properties of Reinforced Solids: Some Theoretical Principles. J. Mech. Phys. Solids, 11, 357-372. http://dx.doi.org/10.1016/0022-5096(63)90036-X

Hutchinson, R. G. (2004). Mechanics of Lattice Materials. Ph.D. thesis, Cambridge University.

Kuznetsov, E. N. (1997). Orthogonal Load Resolution and Statical-Kinematic Stiffness Matrix. International Journal of Solids and Structures, 34, 3657-3671. http://dx.doi.org/10.1016/S0020-7683(96)00229-6

Kuznetsov, E. N. (2000). On the Evaluation of Statical-Kinematic Stiffness Matrix for Under Constrained Structural Systems. International Journal of Solids and Structures, 37(15), 2215-2223. http://dx.doi.org/10.1016/S0020-7683(98)00303-5

Levi-Civita, T., \& Amaldi, U. (1930). Lezioni di Meccanica Razionale (2nd ed.). Vol. 1, Zanichelli, Bologna.

Livesley, R. K. (1975). Matrix Methods of Structural Analysis (2nd ed.). Pergamon international library of science, technology, engineering and social studies. Oxford: Pergamon Press.

Maugin, G. A. (1992). The Thermomechanics of Plasticity and Fracture. Cambridge texts in applied mathematics. Cambridge: Cambridge University Press.

Maxwell, J. C. (1890). On the Calculation of the Equilibrium and Stiffness of Frames. Scientific Papers of J. C. Maxwell, Cambridge University Press, Cambridge, U.K.

McCormac, J. C. (2006). Structural Analysis: Using Classical and Matrix Methods (4th ed.). Wiley.

McGuire, W., Gallagher, R. H., \& Ziemian, R. D. (2000). Matrix Structural Analysis (2nd Ed.). John Wiley and Sons Publishers, New York, New York.

Mohr, O. (1885). Beitrag zur Theorie des Fackwerkes. Der Civilingenieur 31, 289-310. 
Pellegrino, S., \& Calladine, C. R. (1986). Matrix analysis of statically and kinematically indeterminate frameworks. International Journal of Solids and Structures, 22, 409-428. http://dx.doi.org/10.1016/0020-7683(86)90014-4

Pellegrino, S. (1993). Structural Computations with the Singular Value Decomposition of the Equilibrium Matrix. International Journal of Solids and Structures, 34(21) 3025-2035. http://dx.doi.org/10.1016/0020-7683(93)90210-X

Pellegrino, S., \& Calladine, C. R. (1984). Two-Steps Matrix Analysis of Prestressed Cable Nets. Proceeding 3rd Int. Conf. Space Structure, Guildford, Elsevier Applied Science.

Pellegrino, S. (1992). Further Remarks on First-Order Infinitesimal Mechanisms. International Journal of Solids and Structures, 29(17), 2119-2122. http://dx.doi.org/10.1016/0020-7683(92)90060-7

Pellegrino, S. (1990). Analysis of prestressed mechanisms. International Journal of Solids and Structures, 26(12), 1329-1350. http://dx.doi.org/10.1016/0020-7683(90)90082-7

Pestel, E. C., \& Leckie, F. A. (1963). Matrix Methods in Elastomechanics. McGraw-Hill, New York.

Przemieniecki, J. S. (1968). Theory of Matrix Structural Analysis (2nd ed.). New York: McGraw-Hill.

Reddy, J. N. (2002). Energy Principles and Variational Methods in Applied Mechanics. New Jersey: John Wiley.

Renton, J. D. (2002). Elastic Beams and Frames (2nd ed.). Horwood Publishing Limited, England.

Sener, M., Utku, S., \& Wada, B. K., (1994). Geometry Control in Prestressed Adaptive Space Trusses. J. of Smart Materials and Structures, 3, 219. http://dx.doi.org/10.1088/0964-1726/3/2/018

Shan, Z., \& Gokhale, A. M. (2002). Representative volume element for non-uniform micro-structure. $J$. Computational Materials Science, 24, 361-379. http://dx.doi.org/10.1016/S0927-0256(01)00257-9

Strang, G. (1998). Introduction to Linear Algebra (3rd ed.). Wellesley-Cambridge Press.

Tilbert, G. (2002). Deployable Tensegrity Structures for Space Applications. Doctoral thesis, Royal Institute of Technology, Department of Mechanics, Stockholm.

Timoshenko, S. P., \& Young, D. H. (1945). Theory of Structures. McGraw-Hill, New York.

Volokh, K.Yu., \& Vilnay, O. (1997). "Natural", "kinematic" and "elastic" displacements of underconstrained structures. International Journal of Solids and Structures, 34(8), 911-930. http://dx.doi.org/10.1016/S0020-7683(96)00079-0

\section{Appendix}

\section{Stiffness Properties of 2d Rigid-Jointed Lattice Materials Based on Comprehensive Stiffness Analysis}

\section{A.1 Triangular Lattice Material}
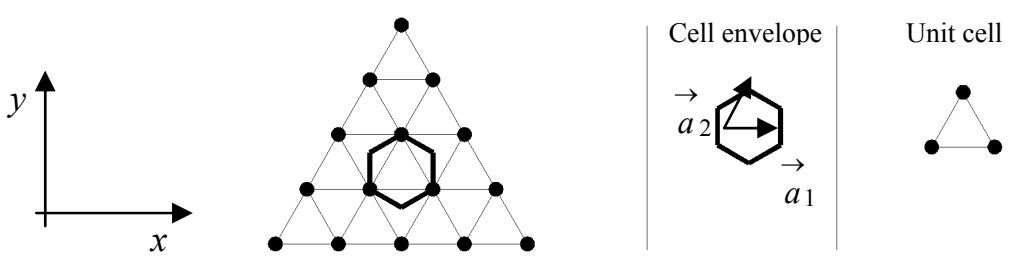

Figure A.1. 2D triangular lattice material

$$
\overline{\mathbf{K}}_{L p}=\frac{\mathbf{K}_{L}}{E}=\bar{\rho}_{L}\left[\begin{array}{ccc}
0.375 & 0.125 & 0 \\
0.125 & 0.375 & 0 \\
0 & 0 & 0.125
\end{array}\right]+\bar{\rho}_{L} \varepsilon_{0}\left[\begin{array}{ccc}
0 & 0 & 0 \\
0 & 0 & 0 \\
0 & 0 & 0
\end{array}\right]
$$




$$
\begin{gathered}
\overline{\mathbf{K}}_{L r}=\frac{\mathbf{K}_{L}}{E}=\bar{\rho}_{L}\left[\begin{array}{ccc}
0.375 & 0.125 & 0 \\
0.125 & 0.375 & 0 \\
0 & 0 & 0.125
\end{array}\right] \\
\rho_{L}=3.464\left(\frac{H}{L}\right)
\end{gathered}
$$

A.2 Lattice Material with Schlafli Symbol of $3^{3} .4^{2}$
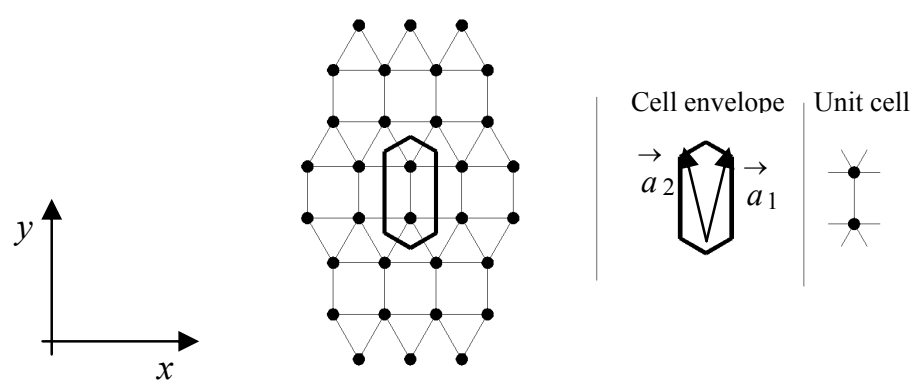

Figure A.2. 2D lattice material with Schlafli symbol of $3^{3} \cdot 4^{2}$

$$
\begin{gathered}
\overline{\mathbf{K}}_{L p}=\frac{\mathbf{K}_{L}}{E}=\bar{\rho}_{L}\left[\begin{array}{ccc}
0.41 & 0.065 & 0 \\
0.065 & 0.418 & 0 \\
0 & 0 & 0
\end{array}\right]+\bar{\rho}_{L} \varepsilon_{0}\left[\begin{array}{ccc}
0 & 0 & 0 \\
0 & 0 & 0 \\
0 & 0 & -0.06
\end{array}\right] \\
\overline{\mathbf{K}}_{L r}=\frac{\mathbf{K}_{L}}{E}=\bar{\rho}_{L}\left[\begin{array}{ccc}
0.41 & 0.065 & 0 \\
0.065 & 0.418 & 0 \\
0 & 0 & 0
\end{array}\right]+\left(\bar{\rho}_{L}\right)^{3}\left[\begin{array}{ccc}
0 & 0 & 0 \\
0 & 0 & 0 \\
0 & 0 & 0.028
\end{array}\right]+\bar{\rho}_{L} \varepsilon_{0}\left[\begin{array}{ccc}
0 & 0 & 0 \\
0 & 0 & 0 \\
0 & 0 & -0.035
\end{array}\right], \\
-\rho_{L}=2.68\left(\frac{H}{L}\right)
\end{gathered}
$$

A.3 Lattice Material with Schlafli Symbol of $3^{4} .6$
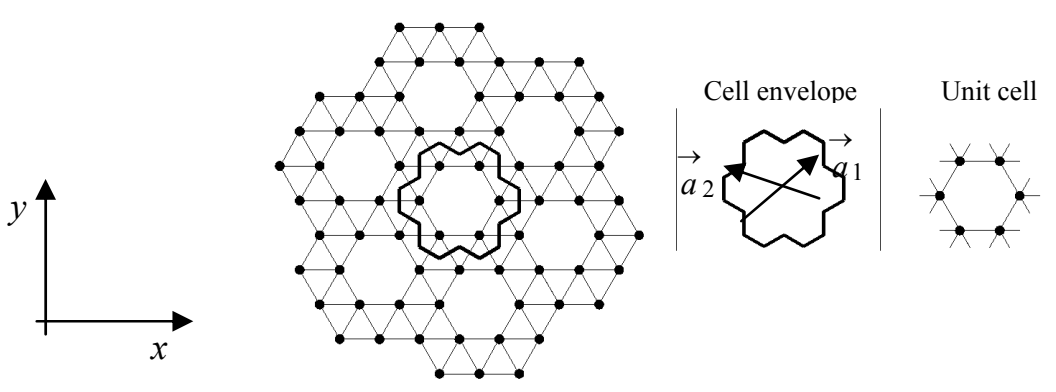

Figure A.3. 2D lattice material with Schlafli symbol of $3^{4} .6$

$$
\overline{\mathbf{K}}_{L p}=\frac{\mathbf{K}_{L}}{E}=\bar{\rho}_{L}\left[\begin{array}{ccc}
0.445 & 0.242 & 0 \\
0.242 & 0.445 & 0 \\
0 & 0 & 0.102
\end{array}\right]+\bar{\rho}_{L} \varepsilon_{0}\left[\begin{array}{ccc}
-0.003 & 0.003 & 0.012 \\
0.003 & -0.003 & -0.012 \\
0.012 & -0.012 & 0.027
\end{array}\right]
$$




$$
\begin{gathered}
\overline{\mathbf{K}}_{L r}=\frac{\mathbf{K}_{L}}{E}=\bar{\rho}_{L}\left[\begin{array}{ccc}
0.445 & 0.242 & 0 \\
0.242 & 0.445 & 0 \\
0 & 0 & 0.102
\end{array}\right]+\left(\bar{\rho}_{L}\right)^{3}\left[\begin{array}{ccc}
0.01 & -0.01 & -0.001 \\
-0.01 & 0.01 & 0.001 \\
-0.001 & 0.001 & 0.012
\end{array}\right] \\
+\rho_{L} \varepsilon_{0}\left[\begin{array}{ccc}
-0.004 & 0.004 & 0.003 \\
0.004 & -0.004 & -0.003 \\
0.003 & -0.003 & 0.005
\end{array}\right] \\
-\rho_{L}=2.474\left(\frac{H}{L}\right)
\end{gathered}
$$

A.4 Double Hexagonal Triangulation (DHT) Lattice Material
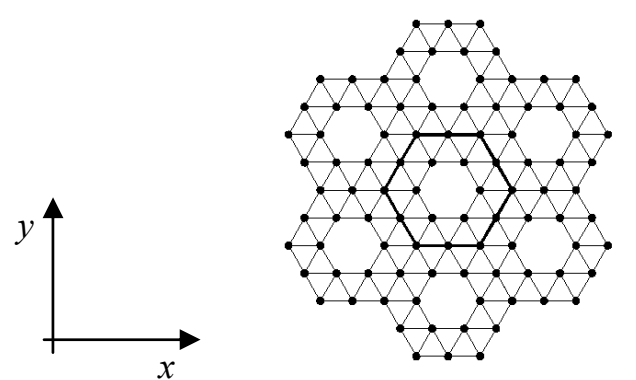

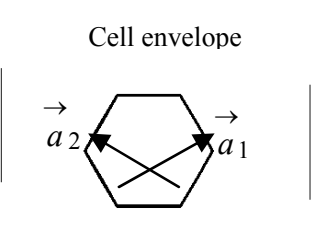

Unit cell

Figure A.4. DHT lattice material

$$
\begin{gathered}
\overline{\mathbf{K}}_{L p}=\frac{\mathbf{K}_{L}}{E}=\bar{\rho}_{L}\left[\begin{array}{ccc}
0.343 & 0.139 & 0 \\
0.139 & 0.343 & 0 \\
0 & 0 & 0.093
\end{array}\right]+\bar{\rho}_{L} \varepsilon_{0}\left[\begin{array}{ccc}
-0.01 & 0.01 & -0.01 \\
0.01 & -0.01 & 0.01 \\
-0.01 & 0.01 & -0.033
\end{array}\right] \\
\overline{\mathbf{K}}_{L r}=\frac{\mathbf{K}_{L}}{E}=\bar{\rho}_{L}\left[\begin{array}{ccc}
0.343 & 0.139 & 0 \\
0.139 & 0.343 & 0 \\
0 & 0 & 0.093
\end{array}\right]+\left(\bar{\rho}_{L}\right)^{3}\left[\begin{array}{ccc}
0.004 & -0.004 & 0 \\
-0.004 & 0.004 & 0 \\
0 & 0 & 0.004
\end{array}\right] \\
+\rho_{L} \varepsilon_{0}\left[\begin{array}{ccc}
-0.006 & 0.006 & -0.003 \\
0.006 & -0.006 & 0.003 \\
-0.003 & 0.0033 & -0.02
\end{array}\right] \\
-\rho_{L}=2.791\left(\frac{H}{L}\right)
\end{gathered}
$$

A.5 Semi-Uni- Braced Square (SUBS) Lattice Material
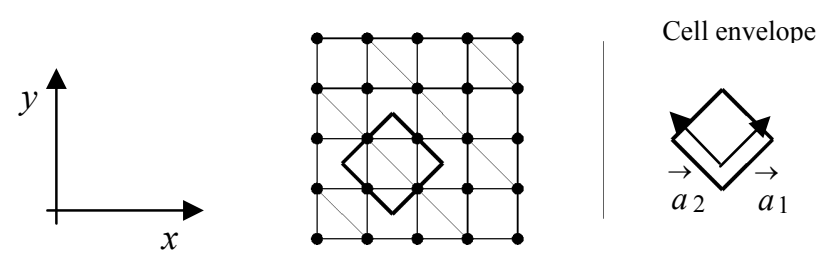

Unit cell

Figure A.5. SUBS lattice material 


$$
\begin{gathered}
\overline{\mathbf{K}}_{L p}=\frac{\mathbf{K}_{L}}{E}=\bar{\rho}_{L}\left[\begin{array}{ccc}
0.435 & 0.065 & -0.065 \\
0.065 & 0.435 & -0.065 \\
-0.065 & -0.065 & 0.065
\end{array}\right]+\bar{\rho}_{L} \varepsilon_{0}\left[\begin{array}{ccc}
-0.092 & 0.092 & 0 \\
0.092 & -0.092 & 0 \\
0 & 0 & -0.092
\end{array}\right] \\
\overline{\mathbf{K}}_{L r}=\frac{\mathbf{K}_{L}}{E}=-\rho_{L}\left[\begin{array}{ccc}
0.435 & 0.065 & -0.065 \\
0.065 & 0.435 & -0.065 \\
-0.065 & -0.065 & 0.065
\end{array}\right]+\left(\bar{\rho}_{L}\right)^{3}\left[\begin{array}{ccc}
0 & 0 & 0 \\
0 & 0 & 0 \\
0 & 0 & 0.013
\end{array}\right] \\
-\rho_{L} \varepsilon_{0}\left[\begin{array}{ccc}
-0.043 & 0.043 & 0 \\
0.043 & -0.043 & 0 \\
0 & 0 & -0.055
\end{array}\right] \\
\rho_{L}=2.707\left(\frac{H}{L}\right)
\end{gathered}
$$

A.6 Triangular-Triangular (TT) Lattice Material

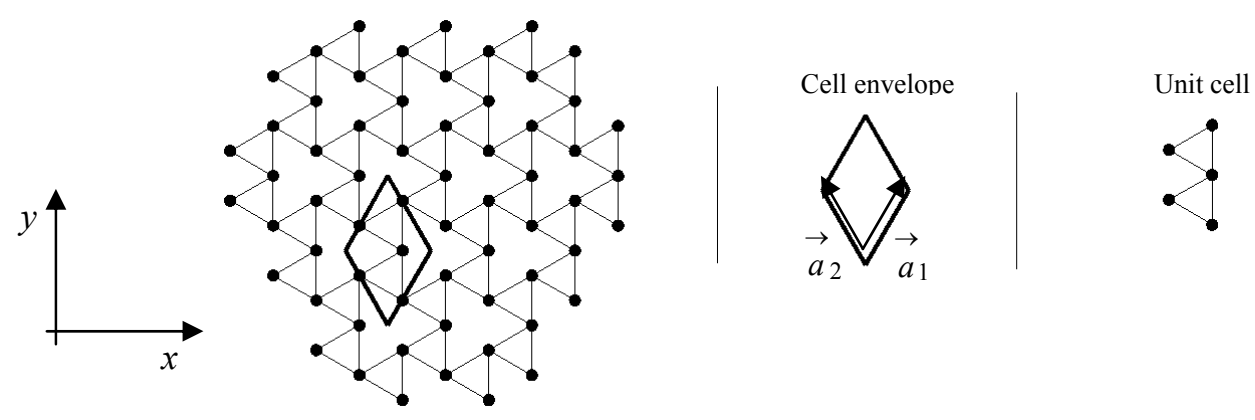

Figure A.6. TT lattice material

$$
\begin{aligned}
& \overline{\mathbf{K}}_{L p}=\frac{\mathbf{K}_{L}}{E}=\bar{\rho}_{L}\left[\begin{array}{ccc}
0.094 & -0.094 & 0 \\
-0.094 & 0.094 & 0 \\
0 & 0 & 0.094
\end{array}\right]+\bar{\rho}_{L} \varepsilon_{0}\left[\begin{array}{ccc}
0.078 & -0.109 & -0.072 \\
-0.109 & 0.14 & 0.054 \\
-0.072 & 0.054 & 0.016
\end{array}\right] \\
& \overline{\mathbf{K}}_{L r}=\frac{\mathbf{K}_{L}}{E}=\bar{\rho}_{L}\left[\begin{array}{ccc}
0.094 & -0.094 & 0 \\
-0.094 & 0.094 & 0 \\
0 & 0 & 0.094
\end{array}\right]+\left(\rho_{L}\right)^{3}\left[\begin{array}{ccc}
0.005 & -0.005 & -0.003 \\
-0.005 & 0.005 & -0.003 \\
-0.003 & 0.003 & 0.002
\end{array}\right] \\
& +\rho_{L} \varepsilon_{0}\left[\begin{array}{ccc}
1.869 & -1.869 & -0.944 \\
-1.869 & 1.869 & 0.944 \\
-1.298 & 1.298 & 0.362
\end{array}\right] \\
& \bar{\rho}_{L}=2.309\left(\frac{H}{L}\right)
\end{aligned}
$$


A.7 Semi-Double Braced Square (SDBS) Lattice Material
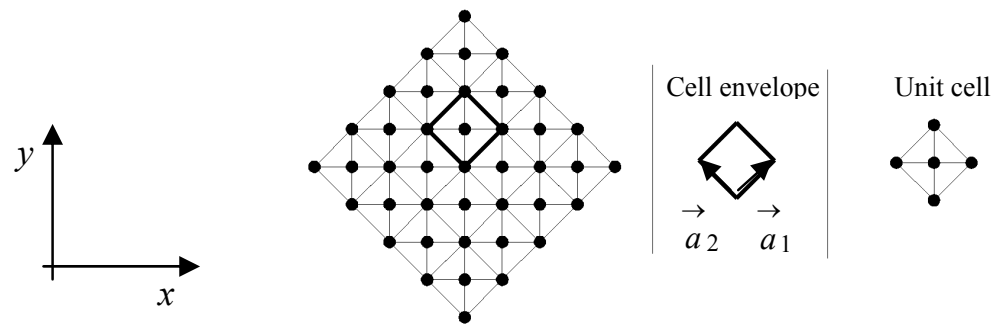

Figure A.7. SDBS lattice material

$$
\begin{aligned}
& \overline{\mathbf{K}}_{L p}=\frac{\mathbf{K}_{L}}{E}=\bar{\rho}_{L}\left[\begin{array}{ccc}
0.396 & 0.104 & 0 \\
0.104 & 0.396 & 0 \\
0 & 0 & 0.104
\end{array}\right]+\bar{\rho}_{L} \varepsilon_{0}\left[\begin{array}{ccc}
-0.146 & 0.146 & 0 \\
0.146 & -0.146 & 0 \\
0 & 0 & -0.073
\end{array}\right] \\
& \overline{\mathbf{K}}_{L r}=\frac{\mathbf{K}_{L}}{E}=\bar{\rho}_{L}\left[\begin{array}{ccc}
0.396 & 0.104 & 0 \\
0.104 & 0.396 & 0 \\
0 & 0 & 0.104
\end{array}\right]+\left(\bar{\rho}_{L}\right)^{3}\left[\begin{array}{ccc}
0.002 & -0.002 & 0 \\
-0.002 & 0.002 & 0 \\
0 & 0 & 0.006
\end{array}\right] \\
& +\bar{\rho}_{L} \varepsilon_{0}\left[\begin{array}{ccc}
-0.088 & 0.088 & 0 \\
0.088 & -0.088 & 0 \\
0 & 0 & -0.044
\end{array}\right] \\
& \bar{\rho}_{L}=3.414\left(\frac{H}{L}\right)
\end{aligned}
$$

A.8 Uni-Braced Square (UBS) Lattice Material
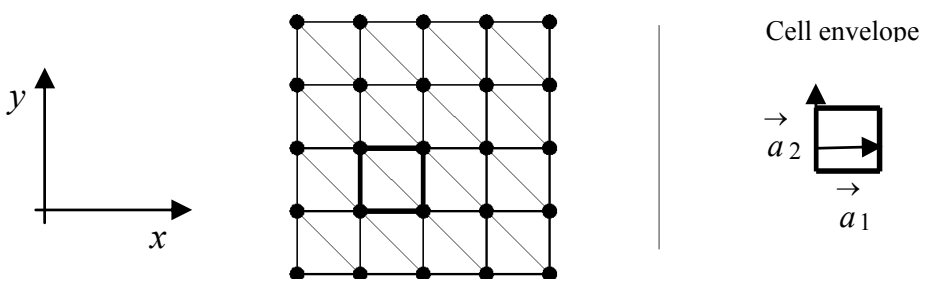

Unit cell

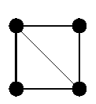

Figure A.8. UBS lattice material

$$
\overline{\mathbf{K}}_{L p}=\frac{\mathbf{K}_{L}}{E}=\bar{\rho}_{L}\left[\begin{array}{ccc}
0.396 & 0.104 & -0.104 \\
0.104 & 0.396 & -0.104 \\
-0.104 & -0.104 & 0.104
\end{array}\right]+\bar{\rho}_{L} \varepsilon_{0}\left[\begin{array}{ccc}
0 & 0 & 0 \\
0 & 0 & 0 \\
0 & 0 & 0
\end{array}\right]
$$




$$
\begin{gathered}
\overline{\mathbf{K}}_{L r}=\frac{\mathbf{K}_{L}}{E}=\bar{\rho}_{L}\left[\begin{array}{ccc}
0.396 & 0.104 & -0.104 \\
0.104 & 0.396 & -0.104 \\
-0.104 & -0.104 & 0.104
\end{array}\right]+\left(\bar{\rho}_{L}\right)^{3}\left[\begin{array}{ccc}
0.002 & -0.002 & 0 \\
-0.002 & 0.002 & 0 \\
0 & 0 & 0.007
\end{array}\right] \\
+\bar{\rho}_{L} \varepsilon_{0}\left[\begin{array}{lll}
0 & 0 & 0 \\
0 & 0 & 0 \\
0 & 0 & 0
\end{array}\right] \\
-\rho_{L}=3.414\left(\frac{H}{L}\right)
\end{gathered}
$$

A.9 Double-Braced Square (DBS) Lattice Material
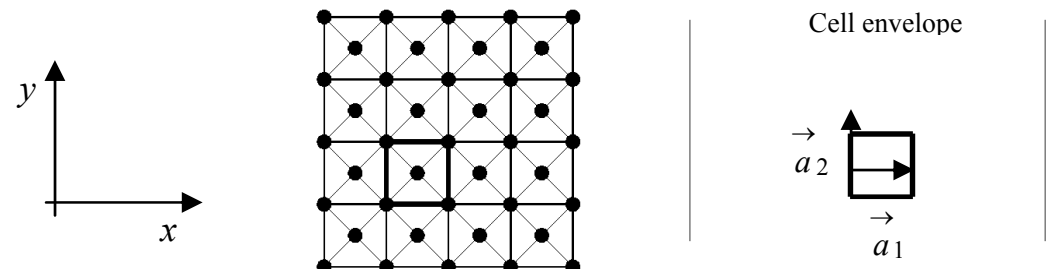

Unit cell

Figure A.9. DBS lattice material

$$
\begin{aligned}
& \overline{\mathbf{K}}_{L p}=\frac{\mathbf{K}_{L}}{E}=\bar{\rho}_{L}\left[\begin{array}{ccc}
0.354 & 0.146 & 0 \\
0.146 & 0.354 & 0 \\
0 & 0 & 0.146
\end{array}\right]+\bar{\rho}_{L} \varepsilon_{0}\left[\begin{array}{ccc}
-0.005 & 0.005 & 0 \\
0.005 & -0.005 & 0 \\
0 & 0 & -0.104
\end{array}\right] \\
& \overline{\mathbf{K}}_{L r}=\frac{\mathbf{K}_{L}}{E}=\bar{\rho}_{L}\left[\begin{array}{ccc}
0.354 & 0.146 & 0 \\
0.146 & 0.354 & 0 \\
0 & 0 & 0.146
\end{array}\right]+\left(\rho_{L}\right)^{3}\left[\begin{array}{ccc}
0.006 & -0.006 & 0 \\
-0.006 & 0.006 & 0 \\
0 & 0 & 0.002
\end{array}\right] \\
& +\bar{\rho}_{L} \varepsilon_{0}\left[\begin{array}{ccc}
-0.031 & 0.031 & 0 \\
0.031 & -0.031 & 0 \\
0 & 0 & -0.062
\end{array}\right] \\
& \bar{\rho}_{L}=4.828\left(\frac{H}{L}\right)
\end{aligned}
$$




\section{A.10. Patched Kagome Lattice Material}

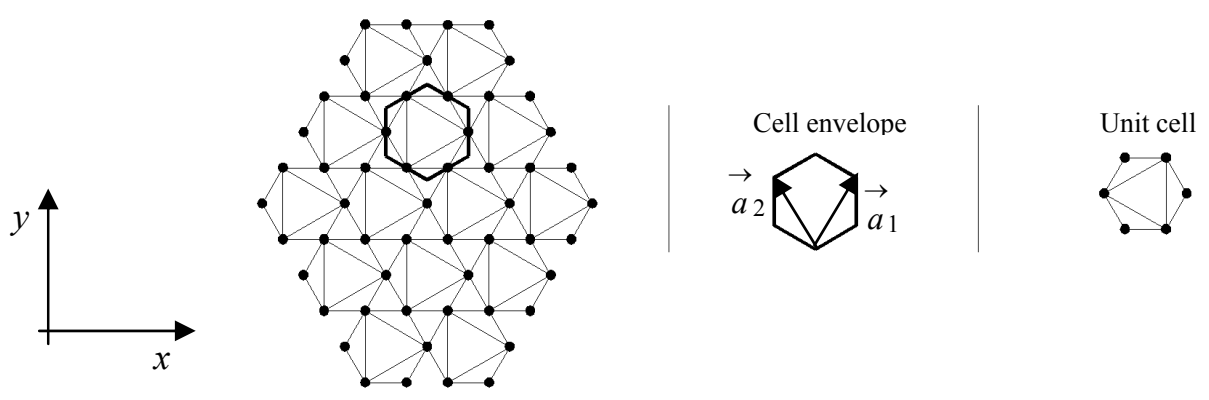

Figure A.10. Patched Kagome lattice material

$$
\begin{gathered}
\overline{\mathbf{K}}_{L p}=\frac{\mathbf{K}_{L}}{E}=\bar{\rho}_{L}\left[\begin{array}{ccc}
0.246 & 0.022 & 0 \\
0.022 & 0.246 & 0 \\
0 & 0 & 0.112
\end{array}\right]+\bar{\rho}_{L} \varepsilon_{0}\left[\begin{array}{ccc}
-0.062 & 0.062 & 0.032 \\
0.062 & -0.062 & -0.032 \\
0.032 & -0.032 & -0.082
\end{array}\right] \\
\overline{\mathbf{K}}_{L r}=\frac{\mathbf{K}_{L}}{E}=\bar{\rho}_{L}\left[\begin{array}{ccc}
0.246 & 0.022 & 0 \\
0.022 & 0.246 & 0 \\
0 & 0 & 0.112
\end{array}\right]+\left(\bar{\rho}_{L}\right)^{3}\left[\begin{array}{ccc}
0.004 & -0.004 & 0 \\
-0.004 & 0.004 & 0 \\
0 & 0 & 0.003
\end{array}\right] \\
+\rho_{L} \varepsilon_{0}\left[\begin{array}{ccc}
-0.036 & 0.036 & 0.02 \\
0.036 & -0.036 & -0.02 \\
0.02 & -0.02 & -0.054
\end{array}\right] \\
- \\
\rho_{L}=3.232\left(\frac{H}{L}\right)
\end{gathered}
$$

A.11 Semi-Hexagonal Triangulation (SHT) Lattice Material
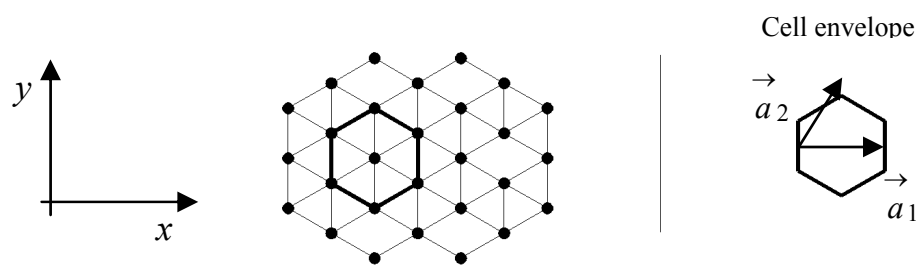

Unit cell

Figure A.11. SHT lattice material

$$
\overline{\mathbf{K}}_{L p}=\frac{\mathbf{K}_{L}}{E}=\bar{\rho}_{L}\left[\begin{array}{ccc}
0.422 & 0.141 & 0 \\
0.141 & 0.197 & 0 \\
0 & 0 & 0.141
\end{array}\right]+-_{L} \varepsilon_{0}\left[\begin{array}{ccc}
-0.092 & 0.092 & 0 \\
0.092 & -0.092 & 0 \\
0 & 0 & -0.013
\end{array}\right]
$$




$$
\begin{gathered}
\overline{\mathbf{K}}_{L r}=\frac{\mathbf{K}_{L}}{E}=\bar{\rho}_{L}\left[\begin{array}{ccc}
0.422 & 0.141 & 0 \\
0.141 & 0.197 & 0 \\
0 & 0 & 0.141
\end{array}\right]+\left(\rho_{L}\right)^{3}\left[\begin{array}{ccc}
0.005 & -0.005 & 0 \\
-0.005 & 0.005 & 0 \\
0 & 0 & 0.013
\end{array}\right] \\
+\rho_{L} \varepsilon_{0}\left[\begin{array}{ccc}
-0.055 & 0.055 & 0 \\
0.055 & -0.055 & 0 \\
0 & 0 & -0.008
\end{array}\right] \\
- \\
\rho_{L}=3.079\left(\frac{H}{L}\right)
\end{gathered}
$$

\section{A.12 Square Lattice Material}

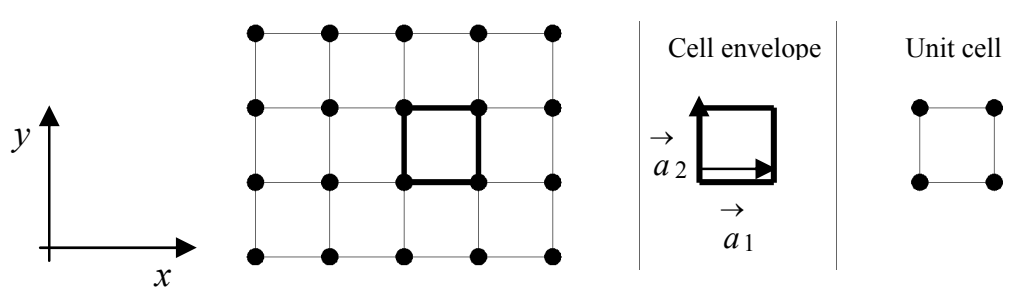

Figure A.12. 2D square lattice material

$$
\begin{aligned}
\overline{\mathbf{K}}_{L p} & =\frac{\mathbf{K}_{L}}{E}=\bar{\rho}_{L}\left[\begin{array}{ccc}
0.5 & 0 & 0 \\
0 & 0.5 & 0 \\
0 & 0 & 0
\end{array}\right]+\bar{\rho}_{L} \varepsilon_{e}\left[\begin{array}{ccc}
0 & 0 & 0 \\
0 & 0 & 0 \\
0 & 0 & 0
\end{array}\right] \\
\overline{\mathbf{K}}_{L r} & =\frac{\mathbf{K}_{L}}{E}=-\rho_{L}\left[\begin{array}{ccc}
0.5 & 0 & 0 \\
0 & 0.5 & 0 \\
0 & 0 & 0
\end{array}\right]+\left(\bar{\rho}_{L}\right)^{3}\left[\begin{array}{ccc}
0 & 0 & 0 \\
0 & 0 & 0 \\
0 & 0 & 0.063
\end{array}\right]+\bar{\rho}_{L} \varepsilon_{e}\left[\begin{array}{lll}
0 & 0 & 0 \\
0 & 0 & 0 \\
0 & 0 & 0
\end{array}\right]
\end{aligned}
$$

